ESAIM: COCV 19 (2013) 754-779

DOI: $10.1051 / \mathrm{cocv} / 2012032$
ESAIM: Control, Optimisation and Calculus of Variations

www.esaim-cocv.org

\title{
ADJOINT METHODS FOR OBSTACLE PROBLEMS AND WEAKLY COUPLED SYSTEMS OF PDE
}

\author{
Filippo Cagnetti ${ }^{1}$, Diogo Gomes ${ }^{1}$ and Hung Vinh Tran ${ }^{2}$
}

\begin{abstract}
The adjoint method, recently introduced by Evans, is used to study obstacle problems, weakly coupled systems, cell problems for weakly coupled systems of Hamilton-Jacobi equations, and weakly coupled systems of obstacle type. In particular, new results about the speed of convergence of some approximation procedures are derived.
\end{abstract}

Mathematics Subject Classification. 35F20, 35F30, 37J50, 49L25.

Received February 29, 2012. Revised August 8, 2012.

Published online June 3, 2013.

\section{INTRODUCTION}

We study the speed of convergence of certain approximations for obstacle problems and weakly coupled systems of Hamilton-Jacobi equations, using the Adjoint Method. This technique, recently introduced by Evans (see [8], and also $[3,14]$ ), is a very successful tool to understand several types of degenerate PDEs. It can be applied, for instance, to Hamilton-Jacobi equations with non convex Hamiltonians, e.g. time dependent (see [8]) and time independent (see [14]), to weak KAM theory (see [3]), and to the infinity Laplacian equation (see [7]). We address here several new applications, and propose some new open questions. Further results, which will not be discussed here, can be found in $[3,8]$.

\subsection{Outline of the paper}

The paper contains four further sections concerning obstacle problems, weakly coupled systems, effective Hamiltonian for weakly coupled systems of Hamilton-Jacobi equations, and weakly coupled systems of obstacle type, respectively. We use a common strategy to study all these problems. Note, however, that each of them presents different challenges, which are described in the corresponding sections. Also, we believe that the applications we present here illustrate how to face the difficulties that can be encountered in the study of other systems of PDEs and related models. In particular, we show how to control singular terms arising from the switching to an obstacle (Lem. 2.3), random switching (Lem. 3.2), or optimal switching (Lem. 5.5).

\footnotetext{
Keywords and phrases. Adjoint methods, cell problems, Hamilton-Jacobi equations, obstacle problems, weakly coupled systems, weak KAM theory.

1 Departamento de Matemática Instituto Superior Técnico, Av. Rovisco Pais, 1049-001 Lisboa, Portugal. cagnetti@math.ist.utl.pt; dgomes@math.ist.utl.pt

2 Department of Mathematics, University of California Berkeley, CA, 94720-3840, U.S.A. tvhung@math.berkeley.edu
} 
In order to clarify our approach, let us give the details of its application to the obstacle problem (see Sect. 2):

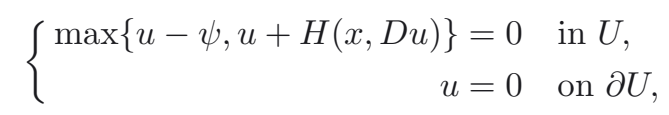

where $\psi: \bar{U} \rightarrow \mathbb{R}$ and $H: \mathbb{R}^{n} \times \bar{U} \rightarrow \mathbb{R}$ are smooth, with $\psi \geq 0$ on $\partial U$. Here and in all the paper, $U$ is an open bounded domain in $\mathbb{R}^{n}$ with smooth boundary, and $n \geq 2$. Moreover, we will denote with $\nu$ the outer unit normal to $\partial U$. This equation arises naturally in optimal control theory, in the study of optimal stopping (see [12]). See also $[1,11]$.

Classically, in order to study (1.1) one first modifies the equation, by adding a perturbation term that penalizes the region where $u>\psi$. Then, a solution is obtained as a limit of the solutions of the penalized problems. More precisely, let $\gamma: \mathbb{R} \rightarrow[0,+\infty)$ be smooth such that

$$
\left\{\begin{array}{c}
\gamma(s)=0 \text { for } s \leq 0, \quad \gamma(s)>0 \text { for } s>0, \\
0<\gamma^{\prime}(s) \leq 1 \text { for } s>0, \text { and } \lim _{s \rightarrow+\infty} \gamma(s)=+\infty,
\end{array}\right.
$$

and define $\gamma^{\varepsilon}: \mathbb{R} \rightarrow[0,+\infty)$ as

$$
\gamma^{\varepsilon}(s):=\gamma\left(\frac{s}{\varepsilon}\right), \text { for all } s \in \mathbb{R}, \quad \text { for all } \varepsilon>0 .
$$

In some of the problems we discuss we also require $\gamma$ to be convex in order to obtain improved results, but that will be pointed out where necessary. For every $\varepsilon>0$, one can introduce the penalized PDE

$$
\left\{\begin{aligned}
u^{\varepsilon}+H\left(x, D u^{\varepsilon}\right)+\gamma^{\varepsilon}\left(u^{\varepsilon}-\psi\right) & =\varepsilon \Delta u^{\varepsilon} \quad \text { in } U \\
u^{\varepsilon} & =0 \quad \text { on } \partial U .
\end{aligned}\right.
$$

To avoid confusion, we stress the fact that here $\gamma^{\varepsilon}\left(u^{\varepsilon}-\psi\right)$ stands for the composition of the function $\gamma^{\varepsilon}$ with $u^{\varepsilon}-\psi$. Unless otherwise stated, we will often simply write $\gamma^{\varepsilon}$ and $\left(\gamma^{\varepsilon}\right)^{\prime}$ to denote $\gamma^{\varepsilon}\left(u^{\varepsilon}-\psi\right)$ and $\left(\gamma^{\varepsilon}\right)^{\prime}\left(u^{\varepsilon}-\psi\right)$, respectively.

Thanks to [12], for every $\varepsilon>0$ there exists a smooth solution $u^{\varepsilon}$ to (1.3). It is also well known that, up to subsequences, $u^{\varepsilon}$ converges uniformly to a viscosity solution $u$ of (1.1) (see also Sect. 2 for further details).

We face here the problem requiring a coercivity assumption on $H$ and a compatibility condition for equation (1.1) (see hypotheses (H2.1) and (H2.2), respectively), and show that the speed of convergence in the general case is $O\left(\varepsilon^{1 / 2}\right)$. Notice that we do not require the Hamiltonian $H$ to be convex in $p$.

Theorem 1.1. Suppose conditions (H2.1) and (H2.2) in Section 2 hold. Then, there exists a positive constant $C$, independent of $\varepsilon$, such that

$$
\left\|u^{\varepsilon}-u\right\|_{L^{\infty}} \leq C \varepsilon^{1 / 2}
$$

The Proof of Theorem 1.1 consists of three steps.

Step I: Preliminary estimates. We first show that

$$
\max _{x \in \bar{U}} \frac{u^{\varepsilon}(x)-\psi(x)}{\varepsilon} \leq C
$$

for some constant $C>0$ independent of $\varepsilon$ (see Lem. 2.2). This allows us to prove that

$$
\left\|u^{\varepsilon}\right\|_{L^{\infty}},\left\|D u^{\varepsilon}\right\|_{L^{\infty}} \leq C
$$

see Proposition 2.1. 
Step II: Adjoint method. We consider the formal linearization of (1.3), and then introduce the correspondent adjoint equation (see Eq. (2.6)). The study of this last equation for different values of the right-hand side allows us to obtain several useful estimates (see Lems. 2.3 and 2.4).

Step III: Conclusion. We conclude the Proof of Theorem 1.1 by showing that

$$
\max _{x \in \bar{U}}\left|u_{\varepsilon}^{\varepsilon}(x)\right| \leq \frac{C}{\varepsilon^{1 / 2}}, \quad \quad u_{\varepsilon}^{\varepsilon}(x):=\frac{\partial u^{\varepsilon}}{\partial \varepsilon}(x),
$$

for some constant $C>0$ independent of $\varepsilon$ (see Lem. 2.5). The most delicate part of the proof of (1.5) consists in controlling the term (see relation $(2.11)$ )

$$
\gamma_{\varepsilon}^{\varepsilon}(s):=\frac{\partial \gamma^{\varepsilon}}{\partial \varepsilon}(s)=-\frac{s}{\varepsilon^{2}} \gamma^{\prime}\left(\frac{s}{\varepsilon}\right), \quad \text { for } s \in \mathbb{R} .
$$

We underline that getting a bound for (1.6) can be extremely hard in general. In this context, this is achieved by differentiating equation (1.3) w.r.t. $\varepsilon$ (see Eq. (2.10)), and then by using inequality (1.4), Lemmas 2.3 and 2.4. This means that we overcome the problem by essentially using the Maximum Principle and the monotonicity of $\gamma^{\varepsilon}$ (see estimates (2.12) and (2.13)). We were not able to obtain such a bound when dealing with homogenization or singular perturbation, where also similar terms appear. We believe it would be very interesting to find the correct way to apply the Adjoint Method in these situations.

In Section 3 we study monotone weakly coupled systems of Hamilton-Jacobi equations

$$
\left\{\begin{array}{l}
c_{11} u_{1}+c_{12} u_{2}+H_{1}\left(x, D u_{1}\right)=0, \\
c_{21} u_{1}+c_{22} u_{2}+H_{2}\left(x, D u_{2}\right)=0,
\end{array} \text { in } U\right.
$$

with boundary conditions $u_{1}=u_{2}=0$ on $\partial U$, by considering the following approximation:

$$
\left\{\begin{array}{l}
c_{11} u_{1}^{\varepsilon}+c_{12} u_{2}^{\varepsilon}+H_{1}\left(x, D u_{1}^{\varepsilon}\right)=\varepsilon \Delta u_{1}^{\varepsilon} \\
c_{21} u_{1}^{\varepsilon}+c_{22} u_{2}^{\varepsilon}+H_{2}\left(x, D u_{2}^{\varepsilon}\right)=\varepsilon \Delta u_{2}^{\varepsilon}
\end{array} \quad \text { in } U\right.
$$

with $u_{1}^{\varepsilon}=u_{2}^{\varepsilon}=0$ on $\partial U$. Under some coupling assumptions on the coefficients (see conditions (H3.2) and (H3.3)), Engler and Lenhart [6], Ishii and Koike [10] prove existence, uniqueness and stability for the viscosity solutions $\left(u_{1}, u_{2}\right)$ of $(1.7)$, but they do not consider any approximation of the system. We observe that these coupling assumptions are similar to monotone conditions of single equations, and play a crucial role in the establishment of the comparison principle and uniqueness result, and thus cannot be removed.

We show that, under the same assumptions of [6], the speed of convergence of $\left(u_{1}^{\varepsilon}, u_{2}^{\varepsilon}\right)$ to $\left(u_{1}, u_{2}\right)$ is $O\left(\varepsilon^{1 / 2}\right)$ (see Thm. 3.5). For the sake of simplicity, we just focus on a system of two equations, but the general case can be treated in a similar way.

Section 4 is devoted to an analog of the cell problem introduced by Lions, Papanicolaou and Varadhan [13]. More precisely, we consider the following quasi-monotone weakly coupled system of Hamilton-Jacobi equations:

$$
\left\{\begin{aligned}
c_{1} u_{1}-c_{1} u_{2}+H_{1}\left(x, D u_{1}\right) & =\bar{H}_{1} \quad \text { in } \mathbb{T}^{n}, \\
-c_{2} u_{1}+c_{2} u_{2}+H_{2}\left(x, D u_{2}\right) & =\bar{H}_{2}
\end{aligned}\right. \text {, }
$$

also called the cell problem. Here $c_{1}$ and $c_{2}$ are positive constants and $H_{1}, H_{2}: \mathbb{T}^{n} \times \mathbb{R}^{n} \rightarrow \mathbb{R}$ are smooth, while $u_{1}, u_{2}: \mathbb{T}^{n} \rightarrow \mathbb{R}$ and $\bar{H}_{1}, \bar{H}_{2} \in \mathbb{R}$ are unknowns. Systems of this type have been studied by Camilli, Loreti and Yamada in [4] and [5], for uniformly convex Hamiltonians in a bounded domain. They arise naturally in optimal control and in large deviation theory for random evolution processes. Under a coercivity-like assumption on $H_{1}, H_{2}$ (see condition (H4.1)), we obtain the following new result.

Theorem 1.2. Assume that (H4.1) holds. Then, there exists a pair of constants $\left(\bar{H}_{1}, \bar{H}_{2}\right)$ such that (1.8) admits a viscosity solution $\left(u_{1}, u_{2}\right) \in C\left(\mathbb{T}^{n}\right)^{2}$. 
One can easily see that the pair $\left(\bar{H}_{1}, \bar{H}_{2}\right)$ is not unique (see Rem. 4.2). Nevertheless, we have the following.

Theorem 1.3. There exists a unique $\mu \in \mathbb{R}$ such that

$$
c_{2} \bar{H}_{1}+c_{1} \bar{H}_{2}=\mu
$$

for every pair $\left(\bar{H}_{1}, \bar{H}_{2}\right) \in \mathbb{R}^{2}$ such that (1.8) admits a viscosity solution $\left(u_{1}, u_{2}\right) \in C\left(\mathbb{T}^{n}\right)^{2}$.

Theorem 1.3 can be rephrased by saying that there exists a unique $\bar{H} \in \mathbb{R}$ such that the system

$$
\left\{\begin{aligned}
c_{1} u_{1}-c_{1} u_{2}+H_{1}\left(x, D u_{1}\right) & =\bar{H} \\
-c_{2} u_{1}+c_{2} u_{2}+H_{2}\left(x, D u_{2}\right) & =\bar{H}
\end{aligned} \quad \text { in } \mathbb{T}^{n},\right.
$$

admits viscosity solutions $u_{1}, u_{2} \in C\left(\mathbb{T}^{n}\right)$, with (see Rem. 4.3)

$$
\bar{H}=\frac{\mu}{c_{1}+c_{2}} .
$$

Thus, this is the analogous to the uniqueness result of the effective Hamiltonian for the single equation case in [13]. Notice that this cell problem is the important basis for the study of homogenization and large time behavior of weakly coupled systems of Hamilton-Jacobi equations.

Besides, we also consider the regularized system

$$
\left\{\begin{array}{l}
\left(c_{1}+\varepsilon\right) u_{1}^{\varepsilon}-c_{1} u_{2}^{\varepsilon}+H_{1}\left(x, D u_{1}^{\varepsilon}\right)=\varepsilon^{2} \Delta u_{1}^{\varepsilon} \\
\left(c_{2}+\varepsilon\right) u_{2}^{\varepsilon}-c_{2} u_{1}^{\varepsilon}+H_{2}\left(x, D u_{2}^{\varepsilon}\right)=\varepsilon^{2} \Delta u_{2}^{\varepsilon}
\end{array} \text { in } \mathbb{T}^{n},\right.
$$

and prove that both $\varepsilon u_{1}^{\varepsilon}$ and $\varepsilon u_{2}^{\varepsilon}$ converge uniformly to $-\bar{H}$ with speed of convergence $O(\varepsilon)$ (see Them. 4.3). We call $\bar{H}$ the effective Hamiltonian of the cell problem for the weakly coupled system of Hamilton-Jacobi equations.

In Section 5, we conclude the paper with the study of weakly coupled systems of obstacle type, namely

$$
\begin{cases}\max \left\{u_{1}-u_{2}-\psi_{1}, u_{1}+H_{1}\left(x, D u_{1}\right)\right\}=0 & \text { in } U, \\ \max \left\{u_{2}-u_{1}-\psi_{2}, u_{2}+H_{2}\left(x, D u_{2}\right)\right\}=0 & \text { in } U,\end{cases}
$$

with boundary conditions $u_{1}=u_{2}=0$ on $\partial U$. Problems of this type appeared in $[2,5]$. Here $H_{1}, H_{2}: \bar{U} \times \mathbb{R}^{n} \rightarrow \mathbb{R}$ and $\psi_{1}, \psi_{2}: \bar{U} \rightarrow \mathbb{R}$ are smooth, with $\psi_{1}, \psi_{2} \geq \alpha>0$.

In this case, although the two equations in (1.11) are coupled just through the difference $u_{1}-u_{2}$ (weakly coupled system), the problem turns out to be considerably more difficult than the corresponding scalar equation (1.1). Indeed, we cannot show now the analogous of estimate (1.4) as in Section 2. For this reason, the hypotheses we require are stronger than in the scalar case. Together with the usual hypotheses of coercivity and compatibility (see conditions (H5.2) and (H5.4)), we have to assume that $H_{1}(x, \cdot)$ and $H_{2}(x, \cdot)$ are convex (see (H5.1)), and we also ask that $D_{x} H_{1}$ and $D_{x} H_{2}$ are bounded (see (H5.3)). Under these hypotheses, that are natural in optimal switching problems, we are able to establish several delicate estimates by crucially employing the adjoint method (Lems. from 5.5 to 5.7), which then yield a rate of convergence (Thm. 5.1).

\section{Obstacle PRoblem}

In this section, we study the following obstacle problem

$$
\left\{\begin{aligned}
\max \{u-\psi, u+H(x, D u)\} & =0 & & \text { in } U \\
u & =0 & & \text { on } \partial U
\end{aligned}\right.
$$

where $\psi: \bar{U} \rightarrow \mathbb{R}$ and $H: \bar{U} \times \mathbb{R}^{n} \rightarrow \mathbb{R}$ are smooth, with $\psi \geq 0$ on $\partial U$. We also assume that 
(H2.1) there exists $\beta>0$ such that

$$
\lim _{|p| \rightarrow+\infty}\left(\beta|H(x, p)|^{2}+D_{x} H(x, p) \cdot p\right)=\lim _{|p| \rightarrow+\infty} \frac{H(x, p)}{|p|}=+\infty \text { uniformly in } x \in \bar{U} ;
$$

(H2.2) there exists a function $\Phi \in C^{2}(U) \cap C^{1}(\bar{U})$ such that $\Phi \leq \psi$ on $\bar{U}, \Phi=0$ on $\partial U$ and

$$
\Phi+H(x, D \Phi)<0 \text { in } \bar{U} .
$$

We observe that in the classical case $H(x, p)=\mathcal{H}(p)+V(x)$ with

$$
\lim _{|p| \rightarrow+\infty} \frac{\mathcal{H}(p)}{|p|}=+\infty
$$

or when $H$ is superlinear in $p$ and $\left|D_{x} H(x, p)\right| \leq C(1+|p|)$, then we immediately have (H2.1). Assumption (H2.2) (stating, in particular, that $\Phi$ is a sub-solution of (2.1)), will be used to derive the existence of solutions of (2.1), and to give a uniform bound for the gradient of solutions of the penalized equation below.

\subsection{The classical approach}

For every $\varepsilon>0$, the penalized equation of (2.1) is given by

$$
\left\{\begin{aligned}
u^{\varepsilon}+H\left(x, D u^{\varepsilon}\right)+\gamma^{\varepsilon}\left(u^{\varepsilon}-\psi\right) & =\varepsilon \Delta u^{\varepsilon} \quad \text { in } U \\
u^{\varepsilon} & =0 \quad \text { on } \partial U
\end{aligned}\right.
$$

where $\gamma^{\varepsilon}$ is defined by (1.2). From [12] it follows that under conditions (H2.1) and (H2.2), for every $\varepsilon>0$ there exists a smooth solution $u^{\varepsilon}$ to $(2.2)$. The first result we establish is a uniform bound for the $C^{1}$-norm of the sequence $\left\{u^{\varepsilon}\right\}$.

Proposition 2.1. There exists a positive constant $C$, independent of $\varepsilon$, such that

$$
\left\|u^{\varepsilon}\right\|_{L^{\infty}},\left\|D u^{\varepsilon}\right\|_{L^{\infty}} \leq C .
$$

In order to prove Proposition 2.1, we need the following fundamental lemma:

Lemma 2.2. There exists a constant $C>0$, independent of $\varepsilon$, such that

$$
\max _{x \in \bar{U}} \gamma^{\varepsilon}\left(u^{\varepsilon}-\psi\right) \leq C, \quad \max _{x \in \bar{U}} \frac{u^{\varepsilon}-\psi}{\varepsilon} \leq C .
$$

Proof. We only need to show that $\max _{x \in \bar{U}} \gamma^{\varepsilon}\left(u^{\varepsilon}-\psi\right) \leq C$, since then the second estimate follows directly by the definition of $\gamma^{\varepsilon}$. Since $u^{\varepsilon}-\psi \leq 0$ on $\partial U$, we have $\max _{x \in \partial U} \gamma^{\varepsilon}\left(u^{\varepsilon}-\psi\right)=0$.

Now, if $\max _{x \in \bar{U}} \gamma^{\varepsilon}\left(u^{\varepsilon}-\psi\right)=0$, then we are done. Thus, let us assume that there exists $x_{1} \in U$ such that $\max _{x \in \bar{U}} \gamma^{\varepsilon}\left(u^{\varepsilon}-\psi\right)=\gamma^{\varepsilon}\left(u^{\varepsilon}-\psi\right)\left(x_{1}\right)>0$. Since $\gamma^{\varepsilon}$ is increasing, we also have $\max _{x \in U}\left(u^{\varepsilon}-\psi\right)=u^{\varepsilon}\left(x_{1}\right)-\psi\left(x_{1}\right)$. Thus, using (2.2), by the Maximum principle

$$
\begin{aligned}
\left(u^{\varepsilon}\left(x_{1}\right)-\psi\left(x_{1}\right)\right)+\gamma^{\varepsilon}\left(u^{\varepsilon}\left(x_{1}\right)-\psi\left(x_{1}\right)\right) & =\varepsilon \Delta u^{\varepsilon}\left(x_{1}\right)-H\left(x_{1}, D u^{\varepsilon}\left(x_{1}\right)\right)-\psi\left(x_{1}\right) \\
& \leq \varepsilon \Delta \psi\left(x_{1}\right)-H\left(x_{1}, D \psi\left(x_{1}\right)\right)-\psi\left(x_{1}\right) .
\end{aligned}
$$

Since $u^{\varepsilon}\left(x_{1}\right)-\psi\left(x_{1}\right)>0$,

$$
\gamma^{\varepsilon}\left(u^{\varepsilon}\left(x_{1}\right)-\psi\left(x_{1}\right)\right) \leq \max _{x \in \bar{U}}(|\Delta \psi|+|H(x, D \psi)|+|\psi(x)|) \leq C,
$$

for any $\varepsilon<1$, and this concludes the proof. 
Proof of Proposition 2.1. Suppose there exists $x_{0} \in U$ such that $u^{\varepsilon}\left(x_{0}\right)=\max _{x \in \bar{U}} u^{\varepsilon}(x)$. Then, since $\Delta u^{\varepsilon}\left(x_{0}\right) \leq 0$ and using the fact that $\gamma^{\varepsilon} \geq 0$

$$
\begin{aligned}
u^{\varepsilon}\left(x_{0}\right) & =\varepsilon \Delta u^{\varepsilon}\left(x_{0}\right)-H\left(x_{0}, 0\right)-\gamma^{\varepsilon}\left(u^{\varepsilon}\left(x_{0}\right)-\psi\left(x_{0}\right)\right) \\
& \leq-H\left(x_{0}, 0\right) \leq \max _{x \in \bar{U}}(-H(x, 0)) \leq C .
\end{aligned}
$$

Let now $x_{1} \in U$ be such that $u^{\varepsilon}\left(x_{1}\right)=\min _{x \in \bar{U}} u^{\varepsilon}\left(x_{1}\right)$. Then, using Lemma 2.2,

$$
\begin{aligned}
u^{\varepsilon}\left(x_{1}\right) & =\varepsilon \Delta u^{\varepsilon}\left(x_{1}\right)-H\left(x_{1}, 0\right)-\gamma^{\varepsilon}\left(u^{\varepsilon}\left(x_{1}\right)-\psi\left(x_{1}\right)\right) \\
& \geq-H\left(x_{1}, 0\right)-\gamma^{\varepsilon}\left(u^{\varepsilon}\left(x_{1}\right)-\psi\left(x_{1}\right)\right) \\
& \geq \min _{x \in \bar{U}}\left(-H(x, 0)-\gamma^{\varepsilon}\left(u^{\varepsilon}(x)-\psi(x)\right)\right) \geq-C .
\end{aligned}
$$

This shows that $\left\|u^{\varepsilon}\right\|_{L^{\infty}}$ is bounded.

To prove that $\left\|D u^{\varepsilon}\right\|_{L^{\infty}}$ is bounded independently of $\varepsilon$, we first need to prove that $\left\|D u^{\varepsilon}\right\|_{L^{\infty}(\partial U)}$ is bounded by constructing appropriate barriers.

Let $\Phi$ be as in (H2.2). For $\varepsilon$ small enough, we have that

$$
\Phi+H(x, D \Phi)+\gamma^{\varepsilon}(\Phi-\psi)<\varepsilon \Delta \Phi,
$$

and $\Phi=0$ on $\partial U$. Therefore, $\Phi$ is a sub-solution of (2.2). By the comparison principle, $u^{\varepsilon} \geq \Phi$ in $U$.

Let now $d(x)=\operatorname{dist}(x, \partial U)$. It is well-known that for some $\delta>0 d \in C^{2}\left(U_{\delta}\right)$ and $|D d|=1$ in $U_{\delta}$, where $U_{\delta}:=\{x \in U: d(x)<\delta\}$. For $\mu>0$ large enough, the uniform bound on $\left\|u^{\varepsilon}\right\|_{L^{\infty}}$ yields $v:=\mu d \geq u^{\varepsilon}$ on $\partial U_{\delta}$. Assumption (H2.1) then implies

$$
v+H(x, D v)+\gamma^{\varepsilon}(v-\psi)-\varepsilon \Delta v \geq H(x, \mu D d)-C \mu \geq 0,
$$

for $\mu$ is sufficiently large. So the comparison principle gives us that $\Phi \leq u^{\varepsilon} \leq v$ in $U_{\delta}$. Thus, since $\nu$ is the outer unit normal to $\partial U$, and $\Phi=u^{\varepsilon}=v=0$ on $\partial U$, we have

$$
\frac{\partial v}{\partial \nu}(x) \leq \frac{\partial u^{\varepsilon}}{\partial \nu}(x) \leq \frac{\partial \Phi}{\partial \nu}(x), \quad \text { for } x \in \partial U .
$$

Hence, we obtain $\left\|D u^{\varepsilon}\right\|_{L^{\infty}(\partial U)} \leq C$.

Next, let us set $w^{\varepsilon}=\frac{\left|D u^{\varepsilon}\right|^{2}}{2}$. By a direct computation one can see that

$$
2\left(1+\left(\gamma^{\varepsilon}\right)^{\prime}\right) w^{\varepsilon}+D_{p} H \cdot D w^{\varepsilon}+D_{x} H \cdot D u^{\varepsilon}-\left(\gamma^{\varepsilon}\right)^{\prime} D u^{\varepsilon} \cdot D \psi=\varepsilon \Delta w^{\varepsilon}-\varepsilon\left|D^{2} u^{\varepsilon}\right|^{2} .
$$

If $\left\|D u^{\varepsilon}\right\|_{L^{\infty}} \leq \max \left(\|D \psi\|_{L^{\infty}},\left\|D u^{\varepsilon}\right\|_{L^{\infty}(\partial U)}\right)$ then we are done.

Otherwise, $\max \left(\|D \psi\|_{L^{\infty}},\left\|D u^{\varepsilon}\right\|_{L^{\infty}(\partial U)}\right)<\left\|D u^{\varepsilon}\right\|_{L^{\infty}}$. We can choose $x_{2} \in U$ such that $w^{\varepsilon}\left(x_{2}\right)=$ $\max _{x \in \bar{U}} w^{\varepsilon}(x)$. Then, using $(2.3)$

$$
\begin{aligned}
\varepsilon\left|D^{2} u^{\varepsilon}\right|^{2}\left(x_{2}\right)= & \varepsilon \Delta w^{\varepsilon}\left(x_{2}\right)-2 w^{\varepsilon}\left(x_{2}\right)-D_{x} H\left(x_{2}, D u^{\varepsilon}\left(x_{2}\right)\right) \cdot D u^{\varepsilon}\left(x_{2}\right) \\
& +\left(\gamma^{\varepsilon}\right)^{\prime}\left(D u^{\varepsilon}\left(x_{2}\right) \cdot D \psi\left(x_{2}\right)-\left|D u^{\varepsilon}\right|^{2}\left(x_{2}\right)\right) \\
& \leq-D_{x} H\left(x_{2}, D u^{\varepsilon}\left(x_{2}\right)\right) \cdot D u^{\varepsilon}\left(x_{2}\right) .
\end{aligned}
$$

Moreover, for $\varepsilon$ sufficiently small we have

$$
\varepsilon\left|D^{2} u^{\varepsilon}\right|^{2}\left(x_{2}\right) \geq 2 \beta \varepsilon^{2}\left|\Delta u^{\varepsilon}\left(x_{2}\right)\right|^{2}=2 \beta\left[u^{\varepsilon}\left(x_{2}\right)+H\left(x_{2}, D u^{\varepsilon}\left(x_{2}\right)\right)+\gamma^{\varepsilon}\left(u^{\varepsilon}\left(x_{2}\right)-\psi\left(x_{2}\right)\right)\right]^{2} .
$$


By Young's inequality,

$$
\begin{aligned}
\varepsilon\left|D^{2} u^{\varepsilon}\right|^{2}\left(x_{2}\right) & \geq \beta\left|H\left(x_{2}, D u^{\varepsilon}\left(x_{2}\right)\right)\right|^{2}-2 \beta\left[u^{\varepsilon}\left(x_{2}\right)+\gamma^{\varepsilon}\left(u^{\varepsilon}\left(x_{2}\right)-\psi\left(x_{2}\right)\right)\right]^{2} \\
& \geq \beta\left|H\left(x_{2}, D u^{\varepsilon}\left(x_{2}\right)\right)\right|^{2}-C_{\beta},
\end{aligned}
$$

for some positive constant $C_{\beta}$, where we used Lemma 2.2 for the last inequality. Collecting (2.4) and (2.5)

$$
\beta\left|H\left(x_{2}, D u^{\varepsilon}\left(x_{2}\right)\right)\right|^{2}+D_{x} H\left(x_{2}, D u^{\varepsilon}\left(x_{2}\right)\right) \cdot D u^{\varepsilon}\left(x_{2}\right) \leq C_{\beta} .
$$

Recalling hypothesis (H2.1), we must have

$$
\left\|D u^{\varepsilon}\right\|_{L^{\infty}}=\left|D u^{\varepsilon}\left(x_{2}\right)\right| \leq C .
$$

Thanks to Proposition 2.1 one can show that, up to subsequences, $u^{\varepsilon}$ converges uniformly to a viscosity solution $u$ of the obstacle problem (2.1).

\subsection{Proof of Theorem 1.1}

We now study the speed of convergence. To prove our theorem we need several steps.

Adjoint method: The formal linearized operator $L^{\varepsilon}: C^{2}(U) \rightarrow C(U)$ corresponding to (2.2) is given by

$$
L^{\varepsilon} z:=\left(1+\left(\gamma^{\varepsilon}\right)^{\prime}\right) z+D_{p} H \cdot D z-\varepsilon \Delta z .
$$

We will now introduce the adjoint PDE corresponding to $L^{\varepsilon}$. Let $x_{0} \in U$ be fixed. We denote by $\sigma^{\varepsilon}$ the solution of:

$$
\left\{\begin{array}{rlrl}
\left(1+\left(\gamma^{\varepsilon}\right)^{\prime}\right) \sigma^{\varepsilon}-\operatorname{div}\left(D_{p} H \sigma^{\varepsilon}\right) & =\varepsilon \Delta \sigma^{\varepsilon}+\delta_{x_{0}}, & & \text { in } U, \\
\sigma^{\varepsilon} & =0, & \text { on } \partial U,
\end{array}\right.
$$

where $\delta_{x_{0}}$ stands for the Dirac measure concentrated in $x_{0}$. In order to show existence and uniqueness of $\sigma^{\varepsilon}$, we have to pass to a further adjoint equation. Let $f \in C(U)$ be fixed. Then, we denote by $v$ the solution to

$$
\left\{\begin{array}{rlrl}
\left(1+\left(\gamma^{\varepsilon}\right)^{\prime}\right) v+D_{p} H \cdot D v & =\varepsilon \Delta v+f, & & \text { in } U, \\
v & =0, & \text { on } \partial U .
\end{array}\right.
$$

When $f \equiv 0$, by using the Maximum Principle one can show that $v \equiv 0$ is the unique solution to (2.7). Thus, by the Fredholm Alternative we infer that (2.6) admits a unique solution $\sigma^{\varepsilon}$. Moreover, one can also prove that $\sigma^{\varepsilon} \in C^{\infty}\left(U \backslash\left\{x_{0}\right\}\right)$. Some additional properties of $\sigma^{\varepsilon}$ are given by the following lemma.

Lemma 2.3 (properties of $\sigma^{\varepsilon}$ ). Let $\nu$ denote the outer unit normal to $\partial U$. Then,

(i) $\sigma^{\varepsilon} \geq 0$ on $\bar{U}$. In particular, $\frac{\partial \sigma^{\varepsilon}}{\partial \nu} \leq 0$ on $\partial U$.

(ii) The following equality holds:

$$
\int_{U}\left(1+\left(\gamma^{\varepsilon}\right)^{\prime}\right) \sigma^{\varepsilon} \mathrm{d} x=1+\varepsilon \int_{\partial U} \frac{\partial \sigma^{\varepsilon}}{\partial \nu} \mathrm{d} S
$$

In particular,

$$
\varepsilon \int_{\partial U}\left|\frac{\partial \sigma^{\varepsilon}}{\partial \nu}\right| \mathrm{d} S \leq 1
$$


Proof. First of all, consider equation (2.7) and observe that

$$
f \geq 0 \Longrightarrow v \geq 0 \text {. }
$$

Indeed, assume $f \geq 0$ and let $\bar{x} \in \bar{U}$ be such that

$$
v(\bar{x})=\min _{x \in \bar{U}} v(x) .
$$

We can assume that $\bar{x} \in U$, since otherwise clearly $v \geq 0$. Then, for every $x \in U$

$$
\left(\left(1+\left(\gamma^{\varepsilon}\right)^{\prime}\right) v(\bar{x})=\varepsilon \Delta v(\bar{x})+f(\bar{x}) \geq 0,\right.
$$

and (2.8) follows, since $1+\left(\gamma^{\varepsilon}\right)^{\prime}>0$.

Now, multiply equation (2.6) by $v$ and integrate by parts, obtaining

$$
\int_{U} f \sigma^{\varepsilon} \mathrm{d} x=v\left(x_{0}\right)
$$

Taking into account (2.8), from last relation we infer that

$$
\int_{U} f \sigma^{\varepsilon} \mathrm{d} x \geq 0 \quad \text { for every } f \geq 0
$$

and this implies $\sigma^{\varepsilon} \geq 0$.

To prove (ii), we integrate (2.6) over $U$, to get

$$
\begin{aligned}
\int_{U}\left(1+\left(\gamma^{\varepsilon}\right)^{\prime}\right) \sigma^{\varepsilon} \mathrm{d} x & =\int_{U} \operatorname{div}\left(D_{p} H \sigma^{\varepsilon}\right) \mathrm{d} x+\varepsilon \int_{U} \Delta \sigma^{\varepsilon} \mathrm{d} x+1 \\
& =\int_{\partial U}\left(D_{p} H \cdot \nu\right) \sigma^{\varepsilon} \mathrm{d} S+\varepsilon \int_{\partial U} \frac{\partial \sigma^{\varepsilon}}{\partial \nu} \mathrm{d} S+1=\varepsilon \int_{\partial U} \frac{\partial \sigma^{\varepsilon}}{\partial \nu} \mathrm{d} S+1,
\end{aligned}
$$

where we used the fact that $\sigma^{\varepsilon}=0$ on $\partial U$.

Using the adjoint equation, we have the following new estimate.

Lemma 2.4. There exists $C>0$, independent of $\varepsilon>0$, such that

$$
\frac{1}{2} \int_{U}\left(1+\left(\gamma^{\varepsilon}\right)^{\prime}\right)\left|D u^{\varepsilon}\right|^{2} \sigma^{\varepsilon} \mathrm{d} x+\varepsilon \int_{U}\left|D^{2} u^{\varepsilon}\right|^{2} \sigma^{\varepsilon} \mathrm{d} x \leq C .
$$

Proof. Multiplying (2.3) by $\sigma^{\varepsilon}$ and integrating by parts, using equation (2.6) we get

$$
\begin{aligned}
\frac{1}{2} \int_{U}\left(1+\left(\gamma^{\varepsilon}\right)^{\prime}\right)\left|D u^{\varepsilon}\right|^{2} \sigma^{\varepsilon} \mathrm{d} x+\varepsilon \int_{U}\left|D^{2} u^{\varepsilon}\right|^{2} \sigma^{\varepsilon} \mathrm{d} x= & -w^{\varepsilon}\left(x_{0}\right)-\int_{U}\left[D_{x} H \cdot D u^{\varepsilon}-\left(\gamma^{\varepsilon}\right)^{\prime} D \psi \cdot D u^{\varepsilon}\right] \sigma^{\varepsilon} \mathrm{d} x \\
& -\varepsilon \int_{\partial U} w^{\varepsilon} \frac{\partial \sigma^{\varepsilon}}{\partial \nu} \mathrm{d} S .
\end{aligned}
$$

Thanks to Lemma 2.3 and Proposition 2.1 (which, in particular, implies $\left.\left\|D_{x} H\left(\cdot, D u^{\varepsilon}(\cdot)\right)\right\|_{L^{\infty}(U)} \leq C\right)$ the conclusion follows.

Relation (2.9) shows that we have a good control of the Hessian $D^{2} u^{\varepsilon}$ in the support of $\sigma^{\varepsilon}$.

We finally have the following result, which immediately implies Theorem 1.1. 
Lemma 2.5. There exists $C>0$, independent of $\varepsilon$, such that

$$
\max _{x \in \bar{U}}\left|u_{\varepsilon}^{\varepsilon}(x)\right| \leq \frac{C}{\varepsilon^{1 / 2}} .
$$

Proof. By standard elliptic estimates, the solution $u^{\varepsilon}$ is smooth in the parameter $\varepsilon$ for $\varepsilon>0$ (see $[8,14]$ for similar arguments). Differentiating (2.2) w.r.t. $\varepsilon$ we get

$$
\left(1+\left(\gamma^{\varepsilon}\right)^{\prime}\right) u_{\varepsilon}^{\varepsilon}+D_{p} H \cdot D u_{\varepsilon}^{\varepsilon}+\gamma_{\varepsilon}^{\varepsilon}=\varepsilon \Delta u_{\varepsilon}^{\varepsilon}+\Delta u^{\varepsilon}, \quad \text { in } U .
$$

In addition, we have $u_{\varepsilon}^{\varepsilon}(x)=0$ for all $x \in \partial U$, since $u^{\varepsilon}(x)=0$ on $\partial U$ for every $\varepsilon$. So, we may assume that there exists $x_{2} \in U$ such that $\left|u_{\varepsilon}^{\varepsilon}\left(x_{2}\right)\right|=\max _{x \in \bar{U}}\left|u_{\varepsilon}^{\varepsilon}(x)\right|$.

Consider the adjoint equation (2.6), and choose $x_{0}=x_{2}$. Multiplying by $\sigma^{\varepsilon}$ both sides of (2.10) and integrating by parts,

$$
u_{\varepsilon}^{\varepsilon}\left(x_{2}\right)=-\int_{U} \gamma_{\varepsilon}^{\varepsilon} \sigma^{\varepsilon} \mathrm{d} x+\int_{U} \Delta u^{\varepsilon} \sigma^{\varepsilon} \mathrm{d} x
$$

Hence,

$$
\left|u_{\varepsilon}^{\varepsilon}\left(x_{2}\right)\right| \leq \int_{U}\left|\gamma_{\varepsilon}^{\varepsilon}\right| \sigma^{\varepsilon} \mathrm{d} x+\int_{U}\left|\Delta u^{\varepsilon}\right| \sigma^{\varepsilon} \mathrm{d} x
$$

By Lemma 2.2,

$$
\left|\gamma_{\varepsilon}^{\varepsilon}\right|=\left|-\frac{u^{\varepsilon}-\psi}{\varepsilon^{2}} \gamma^{\prime}\left(\frac{u^{\varepsilon}-\psi}{\varepsilon}\right)\right|=\left|\frac{u^{\varepsilon}-\psi}{\varepsilon}\left(\gamma^{\varepsilon}\right)^{\prime}\left(u^{\varepsilon}-\psi\right)\right| \leq C\left(\gamma^{\varepsilon}\right)^{\prime} .
$$

Hence, thanks to Lemma 2.3

$$
\int_{U}\left|\gamma_{\varepsilon}^{\varepsilon}\right| \sigma^{\varepsilon} \mathrm{d} x \leq C \int_{U}\left(\gamma^{\varepsilon}\right)^{\prime} \sigma^{\varepsilon} \mathrm{d} x \leq C
$$

while using (2.9)

$$
\begin{aligned}
\int_{U}\left|\Delta u^{\varepsilon}\right| \sigma^{\varepsilon} \mathrm{d} x & \leq\left(\int_{U}\left|\Delta u^{\varepsilon}\right|^{2} \sigma^{\varepsilon} \mathrm{d} x\right)^{1 / 2}\left(\int_{U} \sigma^{\varepsilon} \mathrm{d} x\right)^{1 / 2} \\
& \leq C\left(\int_{U}\left|D^{2} u^{\varepsilon}\right|^{2} \sigma^{\varepsilon} \mathrm{d} x\right)^{1 / 2}\left(\int_{U} \sigma^{\varepsilon} \mathrm{d} x\right)^{1 / 2} \leq \frac{C}{\varepsilon^{1 / 2}} .
\end{aligned}
$$

Thus, by (2.11), (2.13) and (2.14)

$$
\left|u_{\varepsilon}^{\varepsilon}\left(x_{2}\right)\right| \leq \frac{C}{\varepsilon^{1 / 2}}, \text { for } \varepsilon<1
$$

\section{Weakly COUPled systems of Hamilton-Jacobi equations}

We study now the model of monotone weakly coupled systems of Hamilton-Jacobi equations considered by Engler and Lenhart [6], and by Ishii and Koike [10]. For the sake of simplicity, we will just focus on the following system of two equations:

$$
\left\{\begin{array}{l}
c_{11} u_{1}+c_{12} u_{2}+H_{1}\left(x, D u_{1}\right)=0 \\
c_{21} u_{1}+c_{22} u_{2}+H_{2}\left(x, D u_{2}\right)=0
\end{array} \text { in } U\right.
$$

with boundary conditions $u_{1}=u_{2}=0$ on $\partial U$. The general case of more equations can be treated in a similar way.

We assume that the Hamiltonians $H_{1}, H_{2}: \bar{U} \times \mathbb{R}^{n} \rightarrow \mathbb{R}$ are smooth satisfying the following hypotheses. 
(H3.1) There exists $\beta_{1}, \beta_{2}>0$ such that for every $j=1,2$

$$
\lim _{|p| \rightarrow+\infty}\left(\beta_{j}\left|H_{j}(x, p)\right|^{2}+D_{x} H_{j}(x, p) \cdot p\right)=\lim _{|p| \rightarrow+\infty} \frac{H_{j}(x, p)}{|p|}=+\infty \text { uniformly in } x \in \bar{U}
$$

Following [6] and [10], we suppose further that

(H3.2) $c_{12}, c_{21} \leq 0$;

(H3.3) there exists $\alpha>0$ such that $c_{11}+c_{12}, c_{21}+c_{22} \geq \alpha>0$.

We observe that, as a consequence, we also have $c_{11}, c_{22}>0$. Finally, we require that

(H3.4) There exist $\Phi_{1}, \Phi_{2} \in C^{2}(U) \cap C^{1}(\bar{U})$ with $\Phi_{j}=0$ on $\partial U(j=1,2)$, and such that

$$
\begin{cases}c_{11} \Phi_{1}+c_{12} \Phi_{2}+H_{1}\left(x, D \Phi_{1}\right)<0 & \text { in } U, \\ c_{22} \Phi_{2}+c_{21} \Phi_{1}+H_{2}\left(x, D \Phi_{2}\right)<0 & \text { in } U .\end{cases}
$$

Thanks to these conditions, the Maximum Principle can be applied and existence, comparison and uniqueness results hold true, as stated in [6].

We consider now the following regularized system (here $\varepsilon>0$ ):

$$
\left\{\begin{array}{l}
c_{11} u_{1}^{\varepsilon}+c_{12} u_{2}^{\varepsilon}+H_{1}\left(x, D u_{1}^{\varepsilon}\right)=\varepsilon \Delta u_{1}^{\varepsilon} \\
c_{21} u_{1}^{\varepsilon}+c_{22} u_{2}^{\varepsilon}+H_{2}\left(x, D u_{2}^{\varepsilon}\right)=\varepsilon \Delta u_{2}^{\varepsilon}
\end{array} \text { in } U,\right.
$$

with boundary conditions $u_{1}^{\varepsilon}=u_{2}^{\varepsilon}=0$ on $\partial U$.

Conditions (H3.1), (H3.2), and (H3.3) yield existence and uniqueness of the pair of solutions $\left(u_{1}^{\varepsilon}, u_{2}^{\varepsilon}\right)$ in (3.2). Next lemma gives a uniform bound for the $C^{1}$ norm of the sequences $\left\{u_{i}^{\varepsilon}\right\}, i=1,2$. Its proof, which is very similar to that one of Proposition 2.1, is still presented for readers' convenience.

Lemma 3.1. There exists a positive constant $C$, independent of $\varepsilon$, such that

$$
\left\|u_{i}^{\varepsilon}\right\|_{L^{\infty}},\left\|D u_{i}^{\varepsilon}\right\|_{L^{\infty}} \leq C, \quad \text { for } i=1,2 .
$$

Proof. Step I: Bound on $\left\|\mathbf{u}_{\mathbf{j}}^{\varepsilon}\right\|_{\mathbf{L}^{\infty}, \mathbf{j}}=\mathbf{1}, \mathbf{2}$.

First of all observe that $u_{1}^{\varepsilon}=u_{2}^{\varepsilon}=0$ on $\partial U$ for every $\varepsilon$. Thus, it will be sufficient to show that $u_{1}^{\varepsilon}$ and $u_{2}^{\varepsilon}$ are bounded in the interior of $U$. Without loss of generality, we can assume that there exists $\bar{x} \in U$ such that

$$
\max _{\substack{j=1,2 \\ x \in \bar{U}}} u_{j}^{\varepsilon}(x)=u_{1}^{\varepsilon}(\bar{x})
$$

We have

$$
\alpha u_{1}^{\varepsilon}(\bar{x}) \leq c_{11} u_{1}^{\varepsilon}(\bar{x})+c_{12} u_{2}^{\varepsilon}(\bar{x}) \leq-H_{1}(\bar{x}, 0) \leq \max _{x \in \bar{U}}\left(-H_{1}(x, 0)\right),
$$

where we used (H3.3) and equation (3.2). Analogously, if $\widehat{x} \in U$ is such that

$$
\min _{\substack{j=1,2 \\ x \in \bar{U}}} u_{j}^{\varepsilon}(x)=u_{1}^{\varepsilon}(\widehat{x})
$$

then

$$
u_{1}^{\varepsilon}(\widehat{x}) \geq \frac{c_{11}}{c_{11}+c_{12}} u_{1}^{\varepsilon}(\widehat{x})+\frac{c_{12}}{c_{11}+c_{12}} u_{2}^{\varepsilon}(\widehat{x}) \geq-\frac{H_{1}(\widehat{x}, 0)}{c_{11}+c_{12}} \geq \frac{1}{c_{11}+c_{12}} \min _{x \in \bar{U}}\left(-H_{1}(x, 0)\right) .
$$

Concerning the bounds on the gradients, we will argue as in the Proof of Proposition 2.1. 
Step II: Bound on $\left\|\mathbf{D u}_{\mathbf{j}}^{\varepsilon}\right\|_{\mathbf{L}^{\infty}(\partial \mathbf{U})}, \mathbf{j}=\mathbf{1}, \mathbf{2}$.

We now show that

$$
\max _{\substack{j=1,2 \\ x \in \partial U}}\left|D u_{j}^{\varepsilon}(x)\right| \leq C,
$$

for some constant $C$ independent of $\varepsilon$. As it was done in Section 2, we are going to construct appropriate barriers. For $\varepsilon$ small enough, assumption (H3.4) implies that

$$
\begin{cases}c_{11} \Phi_{1}+c_{12} \Phi_{2}+H_{1}\left(x, D \Phi_{1}\right)<\varepsilon \Delta \Phi_{1} & \text { in } U \\ c_{22} \Phi_{2}+c_{21} \Phi_{1}+H_{2}\left(x, D \Phi_{2}\right)<\varepsilon \Delta \Phi_{2} & \text { in } U\end{cases}
$$

and $\Phi_{1}=\Phi_{2}=0$ on $\partial U$. Therefore, $\left(\Phi_{1}, \Phi_{2}\right)$ is a sub-solution of (3.2). By the comparison principle, $u_{j}^{\varepsilon} \geq \Phi_{j}$ in $U, j=1,2$. Let $d(x), \delta$, and $U_{\delta}$ be as in the Proof of Proposition 2.1. For $\mu>0$ large enough, the uniform bounds on $\left\|u_{1}^{\varepsilon}\right\|_{L^{\infty}}$ and $\left\|u_{2}^{\varepsilon}\right\|_{L^{\infty}}$ yield $v:=\mu d \geq u_{j}^{\varepsilon}$ on $\partial U_{\delta}, j=1,2$, so that

$$
\begin{cases}\left(c_{11}+c_{12}\right) v+H_{1}(x, D v)-\varepsilon \Delta v \geq H_{1}(x, \mu D d)-\mu C & \text { in } U, \\ \left(c_{21}+c_{22}\right) v+H_{2}(x, D v)-\varepsilon \Delta v \geq H_{2}(x, \mu D d)-\mu C & \text { in } U .\end{cases}
$$

Now, we have $\Phi_{j}=u_{j}^{\varepsilon}=v=0$ on $\partial U$. Also, thanks to assumption (H3.1), for $\mu>0$ large enough

$$
\begin{cases}\left(c_{11}+c_{12}\right) v+H_{1}(x, D v)-\varepsilon \Delta v \geq 0 & \text { in } U \\ \left(c_{21}+c_{22}\right) v+H_{2}(x, D v)-\varepsilon \Delta v \geq 0 & \text { in } U\end{cases}
$$

that is, the pair $(v, v)$ is a super-solution for the system (3.2). Thus, the comparison principle gives us that $\Phi_{j} \leq u_{j}^{\varepsilon} \leq v$ in $U_{\delta}, j=1,2$. Then, from the fact that $\Phi_{j}=u_{j}^{\varepsilon}=v=0$ on $\partial U$ we get

$$
\frac{\partial v}{\partial \nu}(x) \leq \frac{\partial u_{j}^{\varepsilon}}{\partial \nu}(x) \leq \frac{\partial \Phi_{j}}{\partial \nu}(x), \quad \text { for } x \in \partial U, \quad j=1,2 .
$$

Hence, we obtain $\left\|D u_{j}^{\varepsilon}\right\|_{L^{\infty}(\partial U)} \leq C, j=1,2$.

\section{Step III: Conclusion}

$$
\begin{aligned}
\text { Setting } w_{j}^{\varepsilon}= & \frac{\left|D u_{j}^{\varepsilon}\right|^{2}}{2}, j=1,2 \text {, by a direct computation we have that } \\
& \left\{\begin{array}{l}
2 c_{11} w_{1}^{\varepsilon}+D_{p} H_{1} \cdot D w_{1}^{\varepsilon}+c_{12} D u_{1}^{\varepsilon} \cdot D u_{2}^{\varepsilon}+D_{x} H_{1} \cdot D u_{1}^{\varepsilon}=\varepsilon \Delta w_{1}^{\varepsilon}-\varepsilon\left|D^{2} u_{1}^{\varepsilon}\right|^{2}, \\
2 c_{22} w_{2}^{\varepsilon}+D_{p} H_{2} \cdot D w_{2}^{\varepsilon}+c_{21} D u_{1}^{\varepsilon} \cdot D u_{2}^{\varepsilon}+D_{x} H_{2} \cdot D u_{2}^{\varepsilon}=\varepsilon \Delta w_{2}^{\varepsilon}-\varepsilon\left|D^{2} u_{2}^{\varepsilon}\right|^{2} .
\end{array}\right.
\end{aligned}
$$

Assume now that there exists $\widehat{x} \in U$ such that

$$
\max _{\substack{j=1,2 \\ x \in \bar{U}}} w_{j}^{\varepsilon}(x)=w_{1}^{\varepsilon}(\widehat{x})
$$

Then, we have

$$
\begin{aligned}
\varepsilon\left|D^{2} u_{1}^{\varepsilon}\right|^{2}(\widehat{x}) & =\varepsilon \Delta w_{1}^{\varepsilon}(\widehat{x})-2 c_{11} w_{1}^{\varepsilon}(\widehat{x})-c_{12} D u_{1}^{\varepsilon}(\widehat{x}) \cdot D u_{2}^{\varepsilon}(\widehat{x})-D_{x} H_{1} \cdot D u_{1}^{\varepsilon}(\widehat{x}) \\
& \leq-2\left(c_{11}+c_{12}\right) w_{1}^{\varepsilon}(\widehat{x})-D_{x} H_{1} \cdot D u_{1}^{\varepsilon}(\widehat{x}) \leq-D_{x} H_{1} \cdot D u_{1}^{\varepsilon}(\widehat{x}) .
\end{aligned}
$$

Now, arguing as in the Proof of Proposition 2.1, for $\varepsilon$ sufficiently small

$$
\begin{aligned}
\varepsilon\left|D^{2} u_{1}^{\varepsilon}(\widehat{x})\right|^{2} & \geq 2 \beta_{1} \varepsilon^{2}\left|\Delta u_{1}^{\varepsilon}(\widehat{x})\right|^{2}=2 \beta_{1}\left[c_{11} u_{1}^{\varepsilon}(\widehat{x})+c_{12} u_{2}^{\varepsilon}(\widehat{x})+H_{1}\left(\widehat{x}, D u_{1}^{\varepsilon}(\widehat{x})\right)\right]^{2} \\
& \geq \beta_{1}\left|H_{1}\left(\widehat{x}, D u_{1}^{\varepsilon}(\widehat{x})\right)\right|^{2}-C .
\end{aligned}
$$

Collecting the last two relations we have

$$
\beta_{1}\left|H_{1}\left(\widehat{x}, D u_{1}^{\varepsilon}(\widehat{x})\right)\right|^{2}+D_{x} H_{1}\left(\widehat{x}, D u_{1}^{\varepsilon}(\widehat{x})\right) \cdot D u_{1}^{\varepsilon}(\widehat{x}) \leq C .
$$

Recalling condition (H3.1) the conclusion follows. 
Adjoint method: At this point, we introduce the adjoint of the linearization of system (3.2). Let us emphasize that the adjoint equations we introduce form a system of weakly coupled type, which is very natural in this setting, and create a systematic way to the study of weakly coupled systems. The linearized operator corresponding to $(3.2)$ is

$$
L^{\varepsilon}\left(z_{1}, z_{2}\right):=\left\{\begin{array}{l}
D_{p} H_{1}\left(x, D u_{1}^{\varepsilon}\right) \cdot D z_{1}+c_{11} z_{1}+c_{12} z_{2}-\varepsilon \Delta z_{1} \\
D_{p} H_{2}\left(x, D u_{2}^{\varepsilon}\right) \cdot D z_{2}+c_{22} z_{2}+c_{21} z_{1}-\varepsilon \Delta z_{2}
\end{array}\right.
$$

Let us now identify the adjoint operator $\left(L^{\varepsilon}\right)^{*}$. For every $\nu^{1}, \nu^{2} \in C_{c}^{\infty}(U)$ we have

$$
\begin{aligned}
\left\langle\left(L^{\varepsilon}\right)^{*}\left(\nu^{1}, \nu^{2}\right),\left(z_{1}, z_{2}\right)\right\rangle:= & \left\langle\left(\nu^{1}, \nu^{2}\right), L^{\varepsilon}\left(z_{1}, z_{2}\right)\right\rangle \\
= & \left\langle\nu^{1},\left[L^{\varepsilon}\left(z_{1}, z_{2}\right)\right]_{1}\right\rangle+\left\langle\nu^{2},\left[L^{\varepsilon}\left(z_{1}, z_{2}\right)\right]_{2}\right\rangle \\
= & \int_{U}\left[D_{p} H_{1}\left(x, D u_{1}^{\varepsilon}\right) \cdot D z_{1}+c_{11} z_{1}+c_{12} z_{2}-\varepsilon \Delta z_{1}\right] \nu^{1} \mathrm{~d} x \\
& \quad+\int_{U}\left[D_{p} H_{2}\left(x, D u_{2}^{\varepsilon}\right) \cdot D z_{2}+c_{22} z_{2}+c_{21} z_{1}-\varepsilon \Delta z_{2}\right] \nu^{2} \mathrm{~d} x \\
= & \int_{U}\left[-\operatorname{div}\left(D_{p} H_{1} \nu^{1}\right)+c_{11} \nu^{1}+c_{21} \nu^{2}-\varepsilon \Delta \nu^{1}\right] z_{1} \mathrm{~d} x \\
& \quad+\int_{U}\left[-\operatorname{div}\left(D_{p} H_{2} \nu^{2}\right)+c_{22} \nu^{2}+c_{12} \nu^{1}-\varepsilon \Delta \nu^{2}\right] z_{2} \mathrm{~d} x .
\end{aligned}
$$

Then, the adjoint equations are:

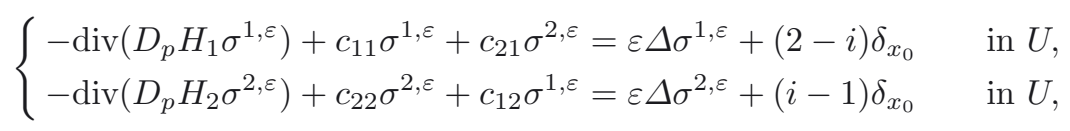

with boundary conditions

$$
\begin{cases}\sigma^{1, \varepsilon}=0 & \text { on } \partial U \\ \sigma^{2, \varepsilon}=0 & \text { on } \partial U\end{cases}
$$

where $i \in\{1,2\}$ and $x_{0} \in U$ will be chosen later. Observe that, once $x_{0}$ is given, the choice $i=1(i=2)$ corresponds to an adjoint system of two equations where a Dirac delta measure concentrated at $x_{0}$ appears only on the right-hand side of the first (second) equation. Existence and uniqueness of $\sigma^{1, \varepsilon}$ and $\sigma^{2, \varepsilon}$ follow by Fredholm alternative, by arguing as in Section 2, and we have $\sigma^{1, \varepsilon}, \sigma^{2, \varepsilon} \in C^{\infty}\left(U \backslash\left\{x_{0}\right\}\right)$. We study now further properties of $\sigma^{1, \varepsilon}$ and $\sigma^{2, \varepsilon}$.

Lemma 3.2 (properties of $\left.\sigma^{1, \varepsilon}, \sigma^{2, \varepsilon}\right)$. Let $\nu$ be the outer unit normal to $\partial U$. Then

(i) $\sigma^{j, \varepsilon} \geq 0$ on $\bar{U}$. In particular, $\frac{\partial \sigma^{j, \varepsilon}}{\partial \nu} \leq 0$ on $\partial U(j=1,2)$.

(ii) The following equality holds:

$$
\sum_{j=1}^{2}\left(\int_{U}\left(c_{j 1}+c_{j 2}\right) \sigma^{j, \varepsilon} \mathrm{d} x-\varepsilon \int_{\partial U} \frac{\partial \sigma^{j, \varepsilon}}{\partial \nu} \mathrm{d} S\right)=1
$$

In particular,

$$
\sum_{j=1}^{2} \int_{U}\left(c_{j 1}+c_{j 2}\right) \sigma^{j, \varepsilon} \mathrm{d} x \leq 1
$$


Proof. First of all, we consider the adjoint of equation (3.4):

$$
\left\{\begin{array}{l}
D_{p} H_{1}\left(x, D u_{1}^{\varepsilon}\right) \cdot D z_{1}+c_{11} z_{1}+c_{12} z_{2}-\varepsilon \Delta z_{1}=f_{1} \\
D_{p} H_{2}\left(x, D u_{2}^{\varepsilon}\right) \cdot D z_{2}+c_{22} z_{2}+c_{21} z_{1}-\varepsilon \Delta z_{2}=f_{2}
\end{array}\right.
$$

where $f_{1}, f_{2} \in C(U)$, with boundary conditions $z_{1}=z_{2}=0$ on $\partial U$. Note that

$$
f_{1}, f_{2} \geq 0 \Longrightarrow \min _{\substack{j=1,2 \\ x \in \bar{U}}} z_{j}(x) \geq 0
$$

Indeed, if the minimum is achieved for some $\bar{x} \in \partial U$, then clearly $z_{1}, z_{2} \geq 0$. Otherwise, assume

$$
\min _{\substack{j=1,2 \\ x \in \bar{U}}} z_{j}(x)=z_{1}(\bar{x})
$$

for some $\bar{x} \in U$. Using condition (H3.2)

$$
\left(c_{11}+c_{12}\right) z_{1}(\bar{x}) \geq c_{11} z_{1}(\bar{x})+c_{12} z_{2}(\bar{x})=\varepsilon \Delta z_{1}(\bar{x})+f_{1}(\bar{x}) \geq 0 .
$$

Thanks to (H3.3), (3.6) follows.

Let us now multiply $(3.4)_{1}$ and $(3.4)_{2}$ by the solutions $z_{1}$ and $z_{2}$ of (3.5). Adding up the relations obtained we have

$$
\int_{U} f_{1} \sigma^{1, \varepsilon} \mathrm{d} x+\int_{U} f_{2} \sigma^{2, \varepsilon} \mathrm{d} x=(2-i) z_{1}\left(x_{0}\right)+(1-i) z_{2}\left(x_{0}\right) .
$$

Thanks to (3.6), from last relation we conclude that

$$
\int_{U} f_{1} \sigma^{1, \varepsilon} \mathrm{d} x+\int_{U} f_{2} \sigma^{2, \varepsilon} \mathrm{d} x \geq 0, \quad \text { for every } f_{1}, f_{2} \geq 0
$$

and this implies that $\sigma^{1, \varepsilon}, \sigma^{2, \varepsilon} \geq 0$. To prove (ii), it is sufficient to integrate equations $(3.4)_{1}$ and (3.4) 2 over $U$, and to add up the two relations obtained.

The proof of the next lemma can be obtained by arguing as in the Proof of Lemma 2.4.

Lemma 3.3. There exists a constant $C>0$, independent of $\varepsilon$, such that

$$
\varepsilon \int_{U}\left|D^{2} u_{1}^{\varepsilon}\right|^{2} \sigma^{1, \varepsilon} \mathrm{d} x+\varepsilon \int_{U}\left|D^{2} u_{2}^{\varepsilon}\right|^{2} \sigma^{2, \varepsilon} \mathrm{d} x \leq C .
$$

We now give the last lemma needed to estimate the speed of convergence. Here we use the notation $u_{j, \varepsilon}^{\varepsilon}(x):=$ $\partial u_{j}^{\varepsilon}(x) / \partial \varepsilon, j=1,2$.

Lemma 3.4. There exists a constant $C>0$, independent of $\varepsilon$, such that

$$
\max _{\substack{j=1,2 \\ x \in \bar{U}}}\left|u_{j, \varepsilon}^{\varepsilon}(x)\right| \leq \frac{C}{\varepsilon^{1 / 2}} .
$$

Proof. Differentiating (3.2) w.r.t $\varepsilon$ we obtain the system

$$
\left\{\begin{array}{l}
c_{11} u_{1, \varepsilon}^{\varepsilon}+c_{12} u_{2, \varepsilon}^{\varepsilon}+D_{p} H_{1} \cdot D u_{1, \varepsilon}^{\varepsilon}=\varepsilon \Delta u_{1, \varepsilon}^{\varepsilon}+\Delta u_{1}^{\varepsilon} \\
c_{21} u_{1, \varepsilon}^{\varepsilon}+c_{22} u_{2, \varepsilon}^{\varepsilon}+D_{p} H_{2} \cdot D u_{2, \varepsilon}^{\varepsilon}=\varepsilon \Delta u_{2, \varepsilon}^{\varepsilon}+\Delta u_{2}^{\varepsilon} .
\end{array}\right.
$$


Since $u_{1, \varepsilon}^{\varepsilon}=u_{2, \varepsilon}^{\varepsilon}=0$ on $\partial U$, we have

$$
\max _{x \in \partial U} u_{1, \varepsilon}^{\varepsilon}(x)=\max _{x \in \partial U} u_{2, \varepsilon}^{\varepsilon}(x)=0 .
$$

Assume now that there exists $\widehat{x} \in U$ such that

$$
\max _{\substack{j=1,2 \\ x \in \bar{U}}}\left|u_{j, \varepsilon}^{\varepsilon}(x)\right|=\left|u_{1, \varepsilon}^{\varepsilon}(\widehat{x})\right|,
$$

and let $\sigma^{1, \varepsilon}, \sigma^{2, \varepsilon}$ be the solutions of system (3.4) with $i=1$ and $x_{0}=\widehat{x}$.

Multiplying equations $(3.7)_{1}$ and $(3.7)_{2}$ by $\sigma^{1, \varepsilon}$ and $\sigma^{2, \varepsilon}$ respectively and adding up, thanks to (3.4) we obtain

$$
u_{1, \varepsilon}^{\varepsilon}(\widehat{x})=\int_{U} \Delta u_{1}^{\varepsilon} \sigma^{1, \varepsilon} \mathrm{d} x+\int_{U} \Delta u_{2}^{\varepsilon} \sigma^{2, \varepsilon} \mathrm{d} x .
$$

Thanks to Lemma 3.3, and repeating the chain of inequalities in (2.14) one can show that

$$
\left|\int_{U} \Delta u_{j}^{\varepsilon} \sigma^{j, \varepsilon} \mathrm{d} x\right| \leq \frac{C}{\varepsilon^{1 / 2}}, \quad j=1,2,
$$

and from this the conclusion follows.

We can now prove the following result on the speed of convergence.

Theorem 3.5. There exists $C>0$, independent of $\varepsilon$, such that

$$
\left\|u_{1}^{\varepsilon}-u_{1}\right\|_{L^{\infty}},\left\|u_{2}^{\varepsilon}-u_{2}\right\|_{L^{\infty}} \leq C \varepsilon^{1 / 2} .
$$

Proof. The theorem is a direct consequence of Lemma 3.4.

\section{Cell problem for Weakly coupled systems of Hamilton-Jacobi equations}

In this section we study the following class of weakly coupled systems of Hamilton-Jacobi equations:

$$
\left\{\begin{aligned}
c_{1} u_{1}-c_{1} u_{2}+H_{1}\left(x, D u_{1}\right) & =\bar{H}_{1} \\
-c_{2} u_{1}+c_{2} u_{2}+H_{2}\left(x, D u_{2}\right) & =\bar{H}_{2}
\end{aligned} \quad \text { in } \mathbb{T}^{n}, \quad \bar{H}_{1}, \bar{H}_{2} \in \mathbb{R},\right.
$$

which is the analog of the cell problem for single equation introduced by Lions, Papanicolaou, and Varadhan [13]. We will assume that $H_{1}, H_{2} \in C^{\infty}\left(\mathbb{T}^{n} \times \mathbb{R}^{n}\right)$, and

(H4.1) there exist $\omega_{1}, \omega_{2}>0$ such that for every $j=1,2$

$$
\lim _{|p| \rightarrow+\infty}\left[\omega_{j}\left|H_{j}(x, p)\right|^{2}+D_{x} H_{j}(x, p) \cdot p-16 n \omega_{j} c_{j}^{2}|p|^{2}\right]=+\infty \text { uniformly in } x \in \mathbb{T}^{n} ;
$$

$(\mathrm{H} 4.2) c_{1}, c_{2}>0$.

It is easy to see that the coefficients of $u_{1}, u_{2}$ in this system do not satisfy the coupling assumptions of the previous section. Indeed, as it happens for the cell problem in the context of weak KAM theory, there is no hope of a uniqueness result for (4.1).

Remark 4.1. The presence of the term $16 n \omega_{j} c_{j}^{2}$ in condition (H4.1) will be justified by later computations. Nevertheless, we observe that (H4.1) is weaker than (H3.1). Indeed, if (H3.1) holds, then for every $\omega_{j}>\beta_{j}$

$$
\begin{aligned}
\lim _{|p| \rightarrow+\infty}\left[\omega_{j}\left|H_{j}(x, p)\right|^{2}+D_{x} H_{j}(x, p) \cdot p-16 n \omega_{j} c_{j}^{2}|p|^{2}\right]= & \lim _{|p| \rightarrow+\infty}\left[\beta_{j}\left|H_{j}(x, p)\right|^{2}+D_{x} H_{j}(x, p) \cdot p+\left(\omega_{j}-\beta_{j}\right)\right. \\
& \left.\times\left|H_{j}(x, p)\right|^{2}-16 n \omega_{j} c_{j}^{2}|p|^{2}\right]=+\infty,
\end{aligned}
$$

uniformly in $x$, and hence (H4.1) is satisfied. 
To find the constants $\bar{H}_{1}, \bar{H}_{2}$ we use the same arguments as in [14]. See also [3,9]. First, for every $\varepsilon>0$, let us consider the following regularized system:

$$
\left\{\begin{array}{l}
\left(c_{1}+\varepsilon\right) u_{1}^{\varepsilon}-c_{1} u_{2}^{\varepsilon}+H_{1}\left(x, D u_{1}^{\varepsilon}\right)=\varepsilon^{2} \Delta u_{1}^{\varepsilon} \\
\left(c_{2}+\varepsilon\right) u_{2}^{\varepsilon}-c_{2} u_{1}^{\varepsilon}+H_{2}\left(x, D u_{2}^{\varepsilon}\right)=\varepsilon^{2} \Delta u_{2}^{\varepsilon}
\end{array} \text { in } \mathbb{T}^{n} .\right.
$$

For every $\varepsilon>0$ fixed, the coefficients of this new system satisfy the coupling assumptions (H3.2) and (H3.3) of the previous section. Thus, (4.2) admits a unique pair of smooth solutions $\left(u_{1}^{\varepsilon}, u_{2}^{\varepsilon}\right)$. In particular, this implies that $u_{1}^{\varepsilon}$ and $u_{2}^{\varepsilon}$ are $\mathbb{T}^{n}$-periodic.

The following result gives some a priori estimates.

Theorem 4.1. There exists $C>0$, independent of $\varepsilon$, such that

$$
\left\|\varepsilon u_{1}^{\varepsilon}\right\|_{L^{\infty}},\left\|\varepsilon u_{2}^{\varepsilon}\right\|_{L^{\infty}},\left\|D u_{1}^{\varepsilon}\right\|_{L^{\infty}},\left\|D u_{2}^{\varepsilon}\right\|_{L^{\infty}} \leq C .
$$

Proof. Our proof is based on the Maximum Principle. Without loss of generality, we may assume that

$$
\max _{\substack{j=1,2 \\ x \in \mathbb{T}^{n}}}\left\{\varepsilon u_{j}^{\varepsilon}(x)\right\}=\varepsilon u_{1}^{\varepsilon}\left(x_{0}^{\varepsilon}\right),
$$

for some $x_{0}^{\varepsilon} \in \mathbb{T}^{n}$. Applying the Maximum Principle to the first equation of (4.2),

$$
\varepsilon u_{1}^{\varepsilon}\left(x_{0}^{\varepsilon}\right) \leq\left(c_{1}+\varepsilon\right) u_{1}^{\varepsilon}\left(x_{0}^{\varepsilon}\right)-c_{1} u_{2}^{\varepsilon}\left(x_{0}^{\varepsilon}\right) \leq-H^{1}\left(x_{0}^{\varepsilon}, 0\right) \leq C,
$$

and this shows the existence of a bound from above for $\varepsilon u_{1}^{\varepsilon}$ and $\varepsilon u_{2}^{\varepsilon}$. Using a similar argument one can show that there is also a bound from below, so that

$$
\left\|\varepsilon u_{1}^{\varepsilon}\right\|_{L^{\infty}},\left\|\varepsilon u_{2}^{\varepsilon}\right\|_{L^{\infty}} \leq C .
$$

The previous inequality allows us to prove a bound for the difference $u_{1}^{\varepsilon}\left(x_{0}^{\varepsilon}\right)-u_{2}^{\varepsilon}\left(x_{0}^{\varepsilon}\right)$. Indeed, thanks to (4.3) and (4.4) we have

$$
\left|u_{1}^{\varepsilon}\left(x_{0}^{\varepsilon}\right)-u_{2}^{\varepsilon}\left(x_{0}^{\varepsilon}\right)\right|=u_{1}^{\varepsilon}\left(x_{0}^{\varepsilon}\right)-u_{2}^{\varepsilon}\left(x_{0}^{\varepsilon}\right) \leq-\frac{1}{c_{1}} H_{1}\left(x_{0}^{\varepsilon}, 0\right)-\frac{\varepsilon}{c_{1}} u_{1}^{\varepsilon}\left(x_{0}^{\varepsilon}\right) \leq C .
$$

In order to find a bound for the gradients, let us set $w_{j}^{\varepsilon}=\frac{\left|D u_{j}^{\varepsilon}\right|^{2}}{2}, j=1,2$. Then, by a direct computation one can see that

$$
\left\{\begin{array}{l}
2\left(c_{1}+\varepsilon\right) w_{1}^{\varepsilon}+D_{p} H_{1} \cdot D w_{1}^{\varepsilon}-c_{1} D u_{1}^{\varepsilon} \cdot D u_{2}^{\varepsilon}+D_{x} H_{1} \cdot D u_{1}^{\varepsilon}=\varepsilon^{2} \Delta w_{1}^{\varepsilon}-\varepsilon^{2}\left|D^{2} u_{1}^{\varepsilon}\right|^{2} \\
2\left(c_{2}+\varepsilon\right) w_{2}^{\varepsilon}+D_{p} H_{2} \cdot D w_{2}^{\varepsilon}-c_{2} D u_{1}^{\varepsilon} \cdot D u_{2}^{\varepsilon}+D_{x} H_{2} \cdot D u_{2}^{\varepsilon}=\varepsilon^{2} \Delta w_{2}^{\varepsilon}-\varepsilon^{2}\left|D^{2} u_{2}^{\varepsilon}\right|^{2}
\end{array} \quad \text { in } \mathbb{T}^{n} .\right.
$$

Without loss of generality, we may assume that there exists $x_{1}^{\varepsilon} \in \mathbb{T}^{n}$ such that

$$
\max _{\substack{j=1,2 \\ x \in \mathbb{T}^{n}}}\left\{w_{j}^{\varepsilon}(x)\right\}=w_{1}^{\varepsilon}\left(x_{1}^{\varepsilon}\right)
$$

Then, by the Maximum Principle

$$
\begin{aligned}
\varepsilon^{2}\left|D^{2} u_{1}^{\varepsilon}\left(x_{1}^{\varepsilon}\right)\right|^{2} & \leq-2\left(c_{1}+\varepsilon\right) w_{1}^{\varepsilon}\left(x_{1}^{\varepsilon}\right)+c_{1} D u_{1}^{\varepsilon}\left(x_{1}^{\varepsilon}\right) \cdot D u_{2}^{\varepsilon}\left(x_{1}^{\varepsilon}\right)-D_{x} H_{1} \cdot D u_{1}^{\varepsilon}\left(x_{1}^{\varepsilon}\right) \\
& \leq-D_{x} H_{1} \cdot D u_{1}^{\varepsilon}\left(x_{1}^{\varepsilon}\right)
\end{aligned}
$$

Moreover, for $\varepsilon$ sufficiently small

$$
\varepsilon^{2}\left|D^{2} u_{1}^{\varepsilon}\left(x_{1}^{\varepsilon}\right)\right|^{2} \geq 2 \omega_{1} \varepsilon^{4}\left(\Delta u_{1}^{\varepsilon}\left(x_{1}^{\varepsilon}\right)\right)^{2}=2 \omega_{1}\left[H_{1}\left(x_{1}^{\varepsilon}, D u_{1}^{\varepsilon}\left(x_{1}^{\varepsilon}\right)\right)+\left(c_{1}+\varepsilon\right) u_{1}^{\varepsilon}\left(x_{1}^{\varepsilon}\right)-c_{1} u_{2}^{\varepsilon}\left(x_{1}^{\varepsilon}\right)\right]^{2} .
$$


Also, thanks to (4.4) and (4.5)

$$
\begin{aligned}
\left|\left(c_{1}+\varepsilon\right) u_{1}^{\varepsilon}\left(x_{1}^{\varepsilon}\right)-c_{1} u_{2}^{\varepsilon}\left(x_{1}^{\varepsilon}\right)\right| \leq & \varepsilon\left|u_{1}^{\varepsilon}\left(x_{1}^{\varepsilon}\right)\right|+c_{1} \mid u_{1}^{\varepsilon}\left(x_{1}^{\varepsilon}\right) \\
& -u_{1}^{\varepsilon}\left(x_{0}^{\varepsilon}\right)\left|+c_{1}\right| u_{2}^{\varepsilon}\left(x_{1}^{\varepsilon}\right)-u_{2}^{\varepsilon}\left(x_{0}^{\varepsilon}\right)\left|+c_{1}\right| u_{1}^{\varepsilon}\left(x_{0}^{\varepsilon}\right)-u_{2}^{\varepsilon}\left(x_{0}^{\varepsilon}\right) \mid \\
\leq & C+c_{1}\left|u_{1}^{\varepsilon}\left(x_{1}^{\varepsilon}\right)-u_{1}^{\varepsilon}\left(x_{0}^{\varepsilon}\right)\right|+c_{1}\left|u_{2}^{\varepsilon}\left(x_{1}^{\varepsilon}\right)-u_{2}^{\varepsilon}\left(x_{0}^{\varepsilon}\right)\right| \\
\leq & C+2 c_{1}\left|D u_{1}^{\varepsilon}\left(x_{1}^{\varepsilon}\right)\right|\left|x_{1}^{\varepsilon}-x_{0}^{\varepsilon}\right| \leq C+2 c_{1} \sqrt{n}\left|D u_{1}^{\varepsilon}\left(x_{1}^{\varepsilon}\right)\right|,
\end{aligned}
$$

where we used the fact that the diameter of $\mathbb{T}^{n}$ is $\sqrt{n}$. Last relation, thanks to (4.7) and Young's inequality, gives that

$$
\begin{aligned}
\varepsilon^{2}\left|D^{2} u_{1}^{\varepsilon}\left(x_{1}^{\varepsilon}\right)\right|^{2} & \geq 2 \omega_{1}\left[H_{1}\left(x_{1}^{\varepsilon}, D u_{1}^{\varepsilon}\left(x_{1}^{\varepsilon}\right)\right)+\left(c_{1}+\varepsilon\right) u_{1}^{\varepsilon}\left(x_{1}^{\varepsilon}\right)-c_{1} u_{2}^{\varepsilon}\left(x_{1}^{\varepsilon}\right)\right]^{2} \\
& \geq \omega_{1}\left|H_{1}\left(x_{1}^{\varepsilon}, D u_{1}^{\varepsilon}\left(x_{1}^{\varepsilon}\right)\right)\right|^{2}-2 \omega_{1}\left[\left(c_{1}+\varepsilon\right) u_{1}^{\varepsilon}\left(x_{1}^{\varepsilon}\right)-c_{1} u_{2}^{\varepsilon}\left(x_{1}^{\varepsilon}\right)\right]^{2} \\
& \geq \omega_{1}\left|H_{1}\left(x_{1}^{\varepsilon}, D u_{1}^{\varepsilon}\left(x_{1}^{\varepsilon}\right)\right)\right|^{2}-2 \omega_{1}\left[C+2 c_{1} \sqrt{n}\left|D u_{1}^{\varepsilon}\left(x_{1}^{\varepsilon}\right)\right|\right]^{2} \\
& \geq \omega_{1}\left|H_{1}\left(x_{1}^{\varepsilon}, D u_{1}^{\varepsilon}\left(x_{1}^{\varepsilon}\right)\right)\right|^{2}-C-16 n \omega_{1} c_{1}^{2}\left|D u_{1}^{\varepsilon}\left(x_{1}^{\varepsilon}\right)\right|^{2} .
\end{aligned}
$$

Using last inequality and (4.6) we have

$$
\omega_{1}\left|H_{1}\left(x_{1}, D u_{1}^{\varepsilon}\left(x_{1}^{\varepsilon}\right)\right)\right|^{2}+D_{x} H_{1} \cdot D u_{1}^{\varepsilon}\left(x_{1}^{\varepsilon}\right)-16 n \omega_{1} c_{1}^{2}\left|D u_{1}^{\varepsilon}\left(x_{1}^{\varepsilon}\right)\right|^{2} \leq C .
$$

Thanks to condition (H4.1), we obtain the conclusion.

In the sequel, all the functions will be regarded as functions defined in the whole $\mathbb{R}^{n}$ and $\mathbb{Z}^{n}$-periodic. Next lemma provides some a priori bounds on $u_{1}^{\varepsilon}$ and $u_{2}^{\varepsilon}$.

Lemma 4.2. There exists a constant $C>0$, independent of $\varepsilon$, such that

$$
\left|u_{1}^{\varepsilon}(x)-u_{1}^{\varepsilon}(y)\right|,\left|u_{2}^{\varepsilon}(x)-u_{2}^{\varepsilon}(y)\right|,\left|u_{1}^{\varepsilon}(x)-u_{2}^{\varepsilon}(y)\right| \leq C, \quad x, y \in \mathbb{R}^{n} .
$$

Proof. The first two inequalities follow from the periodicity of $u_{1}^{\varepsilon}$ and $u_{2}^{\varepsilon}$, and from the fact that $D u_{1}^{\varepsilon}$ and $D u_{2}^{\varepsilon}$ are bounded.

Let us now show the last inequality. As in the previous proof, without loss of generality we may assume that there exists $x_{0}^{\varepsilon} \in \mathbb{T}^{n}$ such that

$$
\max _{\substack{j=1,2 \\ x \in \mathbb{T}^{n}}}\left\{u_{j}^{\varepsilon}(x)\right\}=u_{1}^{\varepsilon}\left(x_{0}^{\varepsilon}\right) .
$$

Combining the second inequality of the lemma with (4.3),

$$
u_{1}^{\varepsilon}(x)-u_{2}^{\varepsilon}(y) \leq u_{1}^{\varepsilon}\left(x_{0}^{\varepsilon}\right)-u_{2}^{\varepsilon}\left(x_{0}^{\varepsilon}\right)+u_{2}^{\varepsilon}\left(x_{0}^{\varepsilon}\right)-u_{2}^{\varepsilon}(y) \leq C, \quad x, y \in \mathbb{R}^{n} .
$$

The proof can be concluded by repeating the same argument for $\min _{\substack{j=1,2 \\ x \in \mathbb{T}^{n}}}\left\{u_{j}^{\varepsilon}(x)\right\}$.

Proof of Theorem 1.2. Thanks to Theorem 4.1 and Lemma 4.2,

$$
\varepsilon u_{i}^{\varepsilon} \rightarrow \bar{C} \quad \text { uniformly in } \mathbb{T}^{n} \text {, for } i=1,2,
$$

for some constant $\bar{C} \in \mathbb{R}$. Furthermore, still up to subsequences,

$$
\left\{\begin{array} { l } 
{ u _ { 1 } ^ { \varepsilon } - \operatorname { m i n } _ { \mathbb { T } ^ { n } } u _ { 1 } ^ { \varepsilon } \rightarrow u _ { 1 } , } \\
{ u _ { 2 } ^ { \varepsilon } - \operatorname { m i n } _ { \mathbb { T } ^ { n } } u _ { 2 } ^ { \varepsilon } \rightarrow u _ { 2 } , }
\end{array} \quad \text { and } \quad \left\{\begin{array}{l}
-\varepsilon u_{1}^{\varepsilon}+c_{1}\left(\min _{\mathbb{T}^{n}} u_{1}^{\varepsilon}-\min _{\mathbb{T}^{n}} u_{2}^{\varepsilon}\right) \rightarrow \bar{H}_{1}, \\
-\varepsilon u_{2}^{\varepsilon}+c_{2}\left(\min _{\mathbb{T}^{n}} u_{2}^{\varepsilon}-\min _{\mathbb{T}^{n}} u_{1}^{\varepsilon}\right) \rightarrow \bar{H}_{2},
\end{array}\right.\right.
$$

uniformly in $\mathbb{T}^{n}$, for some functions $u_{1}, u_{2} \in C\left(\mathbb{T}^{n}\right)$ and some constants $\bar{H}_{1}, \bar{H}_{2} \in \mathbb{R}$. From (4.9) it follows that the functions $\left(u_{1}, u_{2}\right)$ and the constants $\left(\bar{H}_{1}, \bar{H}_{2}\right)$ are such that $(4.1)$ holds, in the viscosity sense. 
Remark 4.2. In general, $\bar{H}_{1}$ and $\bar{H}_{2}$ are not unique. Indeed, let $\left(u_{1}, u_{2}\right)$ be a viscosity solution of (4.1). Then, for every pair of constants $\left(C_{1}, C_{2}\right)$, the pair of functions $\left(\widetilde{u}_{1}, \widetilde{u}_{2}\right)$ where $\widetilde{u}_{1}:=u_{1}+C_{1}$ and $\widetilde{u}_{2}:=u_{2}+C_{2}$ is still a viscosity solution of (4.1), with

$$
\widetilde{H}_{1}:=\bar{H}_{1}+c_{1}\left(C_{1}-C_{2}\right), \quad \widetilde{H}_{2}:=\bar{H}_{2}+c_{2}\left(C_{2}-C_{1}\right),
$$

in place of $\bar{H}_{1}$ and $\bar{H}_{2}$, respectively. Anyway, we have $c_{2} \bar{H}_{1}+c_{1} \bar{H}_{2}=c_{2} \widetilde{H}_{1}+c_{1} \widetilde{H}_{2}$. This suggests that, although $\bar{H}_{1}$ and $\bar{H}_{2}$ may vary, the expression $c_{2} \bar{H}_{1}+c_{1} \bar{H}_{2}$ is unique. Theorem 1.3 shows that this is the case.

Proof of Theorem 1.3. Without loss of generality, we may assume $c_{1}=c_{2}=1$. Suppose, by contradiction, that there exist two pairs $\left(\lambda_{1}, \lambda_{2}\right) \in \mathbb{R}^{2}$ and $\left(\mu_{1}, \mu_{2}\right) \in \mathbb{R}^{2}$, and four functions $u_{1}, u_{2}, \widetilde{u}_{1}, \widetilde{u}_{2} \in C\left(\mathbb{T}^{n}\right)$ such that $\lambda_{1}+\lambda_{2}<\mu_{1}+\mu_{2}$ and

$$
\left\{\begin{aligned}
u_{1}-u_{2}+H_{1}\left(x, D u_{1}\right) & =\lambda_{1} \\
-u_{1}+u_{2}+H_{2}\left(x, D u_{2}\right) & =\lambda_{2}
\end{aligned} \quad \text { in } \mathbb{T}^{n}\right.
$$

and

$$
\left\{\begin{aligned}
\widetilde{u}_{1}-\widetilde{u}_{2}+H_{1}\left(x, D \widetilde{u}_{1}\right) & =\mu_{1} \\
-\widetilde{u}_{1}+\widetilde{u}_{2}+H_{2}\left(x, D \widetilde{u}_{2}\right) & =\mu_{2}
\end{aligned} \text { in } \mathbb{T}^{n} .\right.
$$

By possibly substituting $u_{1}$ and $u_{2}$ with functions $\widehat{u}_{1}:=u_{1}+C_{1}$ and $\widehat{u}_{2}:=u_{2}+C_{2}$, for suitable constants $C_{1}$ and $C_{2}$, we may always assume that $\lambda_{1}<\mu_{1}, \lambda_{2}<\mu_{2}$.

In the same way, by a further substitution $\bar{u}_{1}:=u_{1}+C_{3}, \bar{u}_{2}:=u_{2}+C_{3}$, with $C_{3}>0$ large enough, we may assume that $u_{1}>\widetilde{u}_{1}, u_{2}>\widetilde{u}_{2}$. Then, there exists $\varepsilon>0$ small enough such that

$$
\left\{\begin{array}{l}
(\varepsilon+1) u_{1}-u_{2}+H_{1}\left(x, D u_{1}\right)<(\varepsilon+1) \widetilde{u}_{1}-\widetilde{u}_{2}+H_{1}\left(x, D \widetilde{u}_{1}\right) \\
(\varepsilon+1) u_{2}-u_{1}+H_{2}\left(x, D u_{2}\right)<(\varepsilon+1) \widetilde{u}_{2}-\widetilde{u}_{1}+H_{2}\left(x, D \widetilde{u}_{2}\right)
\end{array} \quad \text { in } \mathbb{T}^{n} .\right.
$$

Observe that the coefficients of the last system satisfy the coupling assumptions (H3.2) and (H3.3). Hence, applying the Comparison Principle in [6] and [10], we conclude that $u_{1}<\widetilde{u}_{1}$ and $u_{2}<\widetilde{u}_{2}$, which gives a contradiction.

Remark 4.3. Multiplying the two convergences in the right in (4.9) by $c_{2}$ and $c_{1}$, respectively, one can see that

$$
-\bar{C}=\bar{H}=\frac{\mu}{c_{1}+c_{2}} .
$$

Here, $\bar{C}$ is defined in (4.8), $\mu$ given by Theorem 1.3, and $\bar{H}$ is the unique constant such that (1.9) has viscosity solutions. We call $\bar{H}$ the effective Hamiltonian of the cell problem for the weakly coupled system of Hamilton-Jacobi equations.

The following is the main theorem of the section. See also [14] for similar results.

Theorem 4.3. There exists a constant $C>0$, independent of $\varepsilon$, such that

$$
\left\|\varepsilon u_{i}^{\varepsilon}+\bar{H}\right\|_{L^{\infty}} \leq C \varepsilon, \quad \text { for } i=1,2 .
$$

Adjoint method: Also in this case, we introduce the adjoint equations associated to the linearization of the original problem. We look for $\sigma^{1, \varepsilon}, \sigma^{2, \varepsilon}$ which are $\mathbb{T}^{n}$-periodic and such that

$$
\begin{cases}-\operatorname{div}\left(D_{p} H_{1} \sigma^{1, \varepsilon}\right)+\left(c_{1}+\varepsilon\right) \sigma^{1, \varepsilon}-c_{2} \sigma^{2, \varepsilon}=\varepsilon^{2} \Delta \sigma^{1, \varepsilon}+\varepsilon(2-i) \delta_{x_{0}} & \text { in } \mathbb{T}^{n} \\ -\operatorname{div}\left(D_{p} H_{2} \sigma^{2, \varepsilon}\right)+\left(c_{2}+\varepsilon\right) \sigma^{2, \varepsilon}-c_{1} \sigma^{1, \varepsilon}=\varepsilon^{2} \Delta \sigma^{2, \varepsilon}+\varepsilon(i-1) \delta_{x_{0}} & \text { in } \mathbb{T}^{n}\end{cases}
$$

where $i \in\{1,2\}$ and $x_{0} \in \mathbb{T}^{n}$ will be chosen later. The argument used in Section 2 gives also in this case existence and uniqueness for $\sigma^{1, \varepsilon}$ and $\sigma^{2, \varepsilon}$. As before, we also have $\sigma^{1, \varepsilon}, \sigma^{2, \varepsilon} \in C^{\infty}\left(\mathbb{T}^{n} \backslash\left\{x_{0}\right\}\right)$. The next two lemmas can be proved in the same ways as Lemmas 3.2 and 3.3, respectively. 
Lemma 4.4 (Properties of $\sigma^{1, \varepsilon}, \sigma^{2, \varepsilon}$ ). The functions $\sigma^{1, \varepsilon}, \sigma^{2, \varepsilon}$ satisfy the following:

(i) $\sigma^{j, \varepsilon} \geq 0$ on $\mathbb{T}^{n} \quad(j=1,2)$;

(ii) Moreover, the following equality holds:

$$
\sum_{j=1}^{2} \int_{\mathbb{T}^{n}} \sigma^{j, \varepsilon} \mathrm{d} x=1
$$

Lemma 4.5. There exists a constant $C>0$, independent of $\varepsilon$, such that

$$
\begin{aligned}
& \varepsilon^{2} \int_{\mathbb{R}^{n}}\left|D^{2} u_{1}^{\varepsilon}\right|^{2} \sigma^{1, \varepsilon} \mathrm{d} x \leq C, \\
& \varepsilon^{2} \int_{\mathbb{R}^{n}}\left|D^{2} u_{2}^{\varepsilon}\right|^{2} \sigma^{2, \varepsilon} \mathrm{d} x \leq C .
\end{aligned}
$$

Finally, next lemma allows us to prove Theorem 4.3.

Lemma 4.6. There exists a constant $C>0$, independent of $\varepsilon$, such that

$$
\max _{\mathbb{T}^{n}}\left|\left(\varepsilon u_{1}^{\varepsilon}\right)_{\varepsilon}\right|, \quad \max _{\mathbb{T}^{n}}\left|\left(\varepsilon u_{2}^{\varepsilon}\right)_{\varepsilon}\right| \leq C .
$$

Proof. Differentiating (4.2) w.r.t. $\varepsilon$,

$$
\left\{\begin{array}{l}
D_{p} H_{1} \cdot D u_{1, \varepsilon}^{\varepsilon}+\left(c_{1}+\varepsilon\right) u_{1, \varepsilon}^{\varepsilon}+u_{1}^{\varepsilon}-c_{1} u_{2, \varepsilon}^{\varepsilon}=\varepsilon^{2} \Delta u_{1, \varepsilon}^{\varepsilon}+2 \varepsilon \Delta u_{1}^{\varepsilon}, \\
D_{p} H_{2} \cdot D u_{2, \varepsilon}^{\varepsilon}+\left(c_{2}+\varepsilon\right) u_{2, \varepsilon}^{\varepsilon}+u_{2}^{\varepsilon}-c_{2} u_{1, \varepsilon}^{\varepsilon}=\varepsilon^{2} \Delta u_{2, \varepsilon}^{\varepsilon}+2 \varepsilon \Delta u_{2}^{\varepsilon},
\end{array}\right.
$$

where we set $u_{j, \varepsilon}^{\varepsilon}:=\partial u_{j}^{\varepsilon} / \partial \varepsilon, j=1,2$. Without loss of generality, we may assume that there exists $x_{2} \in \mathbb{T}^{n}$ such that

$$
\max _{\substack{j=1,2 \\ x \in \mathbb{T}^{n}}}\left(\varepsilon u_{j}^{\varepsilon}(x)\right)_{\varepsilon}=\max _{\substack{j=1,2 \\ x \in \mathbb{T}^{n}}}\left\{\varepsilon u_{j, \varepsilon}^{\varepsilon}(x)+u_{j}^{\varepsilon}(x)\right\}=\varepsilon u_{1, \varepsilon}^{\varepsilon}\left(x_{2}\right)+u_{1}^{\varepsilon}\left(x_{2}\right)
$$

Choosing $x_{0}=x_{2}$ in the adjoint equation (4.10), and repeating the steps in the proof of Theorem 2.5, we get

$$
\varepsilon u_{1, \varepsilon}^{\varepsilon}\left(x_{2}\right)+\int_{\mathbb{T}^{n}} u_{1}^{\varepsilon} \sigma^{1, \varepsilon} \mathrm{d} x+\int_{\mathbb{T}^{n}} u_{2}^{\varepsilon} \sigma^{2, \varepsilon} \mathrm{d} x \leq 2 \varepsilon \int_{\mathbb{T}^{n}}\left|\Delta u_{1}^{\varepsilon}\right| \sigma^{1, \varepsilon} \mathrm{d} x+2 \varepsilon \int_{\mathbb{T}^{n}}\left|\Delta u_{2}^{\varepsilon}\right| \sigma^{2, \varepsilon} \mathrm{d} x \leq C,
$$

where the latter inequality follows by repeating the chain of inequalities in (2.14) and thanks to Lemma 4.5. Using Lemma 4.2 and property (ii) of Lemma 4.4 we have

$$
\begin{aligned}
\left|\int_{\mathbb{T}^{n}} u_{1}^{\varepsilon}(x) \sigma^{1, \varepsilon} \mathrm{d} x+\int_{\mathbb{T}^{n}} u_{2}^{\varepsilon}(x) \sigma^{2, \varepsilon} \mathrm{d} x-u_{1}^{\varepsilon}\left(x_{2}\right)\right|= & \mid \int_{\mathbb{T}^{n}}\left(u_{1}^{\varepsilon}(x)-u_{1}^{\varepsilon}\left(x_{2}\right)\right) \sigma^{1, \varepsilon} \mathrm{d} x \\
& +\int_{\mathbb{T}^{n}}\left(u_{2}^{\varepsilon}(x)-u_{1}^{\varepsilon}\left(x_{2}\right)\right) \sigma^{2, \varepsilon} \mathrm{d} x \mid \leq C .
\end{aligned}
$$

In view of the previous inequality, (4.11) becomes

$$
\varepsilon u_{1, \varepsilon}^{\varepsilon}\left(x_{2}\right)+u_{1}^{\varepsilon}\left(x_{2}\right) \leq C,
$$

thus giving the bound from above. The same argument, applied to $\min _{\substack{j=1,2 \\ x \in \mathbb{T}^{n}}}\left(\varepsilon u_{j}^{\varepsilon}(x)\right)_{\varepsilon}$, allows to prove the bound from below.

Proof of Theorem 4.3. The theorem immediately follows by using Lemma 4.6. 
Remark 4.4. (i). In order to achieve existence and uniqueness of the effective Hamiltonian $\bar{H}$ one can require either (H4.1) or the usual coercive assumption (i.e. $H_{1}, H_{2}$ are uniformly coercive in $p$ ). Indeed one can consider the regularized system

$$
\left\{\begin{array}{l}
\left(c_{1}+\varepsilon\right) u_{1}^{\varepsilon}-c_{1} u_{2}^{\varepsilon}+H_{1}\left(x, D u_{1}^{\varepsilon}\right)=0 \\
\left(c_{2}+\varepsilon\right) u_{2}^{\varepsilon}-c_{2} u_{1}^{\varepsilon}+H_{2}\left(x, D u_{2}^{\varepsilon}\right)=0
\end{array} \text { in } \mathbb{T}^{n}\right.
$$

and derive the results similarly to what we did above by using the coercivity of $H_{1}, H_{2}$. We require (H4.1) in order to get the speed of convergence as in Theorem 4.3.

(ii) By using the same arguments, we can show that for any $P \in \mathbb{R}^{n}$, there exist a pair of constants $\left(\bar{H}_{1}(P), \bar{H}_{2}(P)\right)$ such that the system

$$
\left\{\begin{array}{l}
c_{1} u_{1}-c_{1} u_{2}+H_{1}\left(x, P+D u_{1}\right)=\bar{H}_{1}(P) \\
c_{2} u_{2}-c_{2} u_{1}+H_{2}\left(x, P+D u_{2}\right)=\bar{H}_{2}(P)
\end{array} \quad \text { in } \mathbb{T}^{n},\right.
$$

admits a solution $\left(u_{1}(\cdot, P), u_{2}(\cdot, P)\right) \in C\left(\mathbb{T}^{n}\right)^{2}$. Moreover $\bar{H}(P)$, the effective Hamiltonian, is unique and

$$
\bar{H}(P)=\frac{c_{2} \bar{H}_{1}(P)+c_{1} \bar{H}_{2}(P)}{c_{1}+c_{2}} .
$$

\section{WEAKLY COUPLED SYSTEMS OF OBSTACLE TYPE}

In this last section we apply the Adjoint Method to weakly coupled systems of obstacle type. Let $H_{1}, H_{2}$ : $\bar{U} \times \mathbb{R}^{n} \rightarrow \mathbb{R}$ be smooth Hamiltonians, and let $\psi_{1}, \psi_{2}: \bar{U} \rightarrow \mathbb{R}$ be smooth functions describing the obstacles. We assume that there exists $\alpha>0$ such that

$$
\psi_{1}, \psi_{2} \geq \alpha \quad \text { in } \bar{U}
$$

and consider the system

$$
\begin{cases}\max \left\{u_{1}-u_{2}-\psi_{1}, u_{1}+H_{1}\left(x, D u_{1}\right)\right\}=0 & \text { in } U, \\ \max \left\{u_{2}-u_{1}-\psi_{2}, u_{2}+H_{2}\left(x, D u_{2}\right)\right\}=0 & \text { in } U,\end{cases}
$$

with boundary conditions $\left.u_{1}\right|_{\partial U}=\left.u_{2}\right|_{\partial U}=0$. We observe that (5.1) guarantees the compatibility of the boundary conditions, since $\psi_{1}, \psi_{2}>0$ on $\partial U$.

Although the two equations in (5.2) are coupled just through the difference $u_{1}-u_{2}$, this problem turns out to be more difficult that the correspondent scalar equation (2.1) studied in Section 2. For this reason, the hypotheses we require now are stronger. We assume that

(H5.1) $H_{j}(x, \cdot)$ is convex for every $x \in \bar{U}, j=1,2$.

(H5.2) Superlinearity in $p$ :

$$
\lim _{|p| \rightarrow \infty} \frac{H_{j}(x, p)}{|p|}=+\infty \quad \text { uniformly in } x, \quad j=1,2 .
$$

(H5.3) $\left|D_{x} H_{j}(x, p)\right| \leq C$ for each $(x, p) \in \bar{U} \times \mathbb{R}^{n}, j=1,2$.

(H5.4) There exist $\Phi_{1}, \Phi_{2} \in C^{2}(U) \cap C^{1}(\bar{U})$ with $\Phi_{j}=0$ on $\partial U(j=1,2),-\psi_{2} \leq \Phi_{1}-\Phi_{2} \leq \psi_{1}$, and such that

$$
\Phi_{j}+H_{j}\left(x, D \Phi_{j}\right)<0 \quad \text { in } \bar{U} \quad(j=1,2) .
$$

Let $\varepsilon>0$ and let $\gamma^{\varepsilon}: \mathbb{R} \rightarrow[0,+\infty)$ be the function defined by (1.2). We make in this section the additional assumption that $\gamma$ is convex. We approximate (5.2) by the following system

$$
\left\{\begin{array}{l}
u_{1}^{\varepsilon}+H_{1}\left(x, D u_{1}^{\varepsilon}\right)+\gamma^{\varepsilon}\left(u_{1}^{\varepsilon}-u_{2}^{\varepsilon}-\psi_{1}\right)=\varepsilon \Delta u_{1}^{\varepsilon} \quad \text { in } U \\
u_{2}^{\varepsilon}+H_{2}\left(x, D u_{2}^{\varepsilon}\right)+\gamma^{\varepsilon}\left(u_{2}^{\varepsilon}-u_{1}^{\varepsilon}-\psi_{2}\right)=\varepsilon \Delta u_{2}^{\varepsilon} \quad \text { in } U
\end{array}\right.
$$

We are now ready to state the main result of the section. 
Theorem 5.1. There exists a positive constant $C$, independent of $\varepsilon$, such that

$$
\left\|u_{i}^{\varepsilon}-u_{i}\right\|_{L^{\infty}} \leq C \varepsilon^{1 / 2}, \quad \text { for } i=1,2 .
$$

In order to prove the theorem we need several lemmas. In the sequel, we shall use the notation

$$
\theta_{1}^{\varepsilon}:=u_{1}^{\varepsilon}-u_{2}^{\varepsilon}-\psi_{1}, \quad \theta_{2}^{\varepsilon}:=u_{2}^{\varepsilon}-u_{1}^{\varepsilon}-\psi_{2} .
$$

The linearized operator corresponding to (5.3) is

$$
L^{\varepsilon}\left(z_{1}, z_{2}\right):=\left\{\begin{array}{l}
z_{1}+D_{p} H_{1}\left(x, D u_{1}^{\varepsilon}\right) \cdot D z_{1}+\left.\left(\gamma^{\varepsilon}\right)^{\prime}\right|_{\theta_{1}^{\varepsilon}}\left(z_{1}-z_{2}\right)-\varepsilon \Delta z_{1}, \\
z_{2}+D_{p} H_{2}\left(x, D u_{2}^{\varepsilon}\right) \cdot D z_{2}+\left.\left(\gamma^{\varepsilon}\right)^{\prime}\right|_{\theta_{2}^{\varepsilon}}\left(z_{2}-z_{1}\right)-\varepsilon \Delta z_{2} .
\end{array}\right.
$$

Then, the adjoint equations are:

$$
\begin{cases}\left(1+\left.\left(\gamma^{\varepsilon}\right)^{\prime}\right|_{\theta_{1}^{\varepsilon}}\right) \sigma^{1, \varepsilon}-\operatorname{div}\left(D_{p} H_{1} \sigma^{1, \varepsilon}\right)-\left.\left(\gamma^{\varepsilon}\right)^{\prime}\right|_{\theta_{2}^{\varepsilon}} \sigma^{2, \varepsilon}=\varepsilon \Delta \sigma^{1, \varepsilon}+(2-i) \delta_{x_{0}} & \text { in } U, \\ \left(1+\left.\left(\gamma^{\varepsilon}\right)^{\prime}\right|_{\theta_{2}^{\varepsilon}}\right) \sigma^{2, \varepsilon}-\operatorname{div}\left(D_{p} H_{2} \sigma^{2, \varepsilon}\right)-\left.\left(\gamma^{\varepsilon}\right)^{\prime}\right|_{\theta_{1}^{\varepsilon}} \sigma^{1, \varepsilon}=\varepsilon \Delta \sigma^{2, \varepsilon}+(i-1) \delta_{x_{0}} & \text { in } U,\end{cases}
$$

with boundary conditions

$$
\begin{cases}\sigma^{1, \varepsilon}=0 & \text { on } \partial U \\ \sigma^{2, \varepsilon}=0 & \text { on } \partial U\end{cases}
$$

where $i \in\{1,2\}$ and $x_{0} \in U$ will be chosen later. By repeating what was done in Section 2, we get the existence and uniqueness of $\sigma^{1, \varepsilon}$ and $\sigma^{2, \varepsilon}$ by Fredholm alternative. Furthermore, $\sigma^{1, \varepsilon}$ and $\sigma^{2, \varepsilon}$ are well defined and $\sigma^{1, \varepsilon}, \sigma^{2, \varepsilon} \in C^{\infty}\left(U \backslash\left\{x_{0}\right\}\right)$. In order to derive further properties of $\sigma^{1, \varepsilon}$ and $\sigma^{2, \varepsilon}$, we need the following useful formulas.

Lemma 5.2. For every $\varphi_{1}, \varphi_{2} \in C^{2}(\bar{U})$ we have

$$
\begin{aligned}
(2-i) \varphi_{1}\left(x_{0}\right)= & -\varepsilon \int_{\partial U} \frac{\partial \sigma^{1, \varepsilon}}{\partial \nu} \varphi_{1} \mathrm{~d} S-\left.\int_{U}\left(\gamma^{\varepsilon}\right)^{\prime}\right|_{\theta_{2}^{\varepsilon}} \varphi_{1} \sigma^{2, \varepsilon} \mathrm{d} x \\
& +\int_{U}\left[\left(1+\left.\left(\gamma^{\varepsilon}\right)^{\prime}\right|_{\theta_{1}^{\varepsilon}}\right) \varphi_{1}+D_{p} H_{1} \cdot D \varphi_{1}-\varepsilon \Delta \varphi_{1}\right] \sigma^{1, \varepsilon} \mathrm{d} x,
\end{aligned}
$$

and

$$
\begin{aligned}
(i-1) \varphi_{2}\left(x_{0}\right)= & -\varepsilon \int_{\partial U} \frac{\partial \sigma^{2, \varepsilon}}{\partial \nu} \varphi_{2} \mathrm{~d} S-\left.\int_{U}\left(\gamma^{\varepsilon}\right)^{\prime}\right|_{\theta_{1}^{\varepsilon}} \varphi_{2} \sigma^{1, \varepsilon} \mathrm{d} x \\
& +\int_{U}\left[\left(1+\left.\left(\gamma^{\varepsilon}\right)^{\prime}\right|_{\theta_{2}^{\varepsilon}}\right) \varphi_{2}+D_{p} H_{2} \cdot D \varphi_{2}-\varepsilon \Delta \varphi_{2}\right] \sigma^{2, \varepsilon} \mathrm{d} x,
\end{aligned}
$$

where $\nu$ is the outer unit normal to $\partial U$.

Proof. The conclusion follows by simply multiplying by $\varphi_{j}(j=1,2)$ the two equations in (5.4) and integrating by parts.

From the previous lemma, the analogous of Lemma 2.3 follows.

Lemma 5.3 (properties of $\left.\sigma^{1, \varepsilon}, \sigma^{2, \varepsilon}\right)$. Let $\nu$ be the outer unit normal to $\partial U$. Then

(i) $\sigma^{j, \varepsilon} \geq 0$ on $\bar{U}$. In particular, $\frac{\partial \sigma^{j, \varepsilon}}{\partial \nu} \leq 0$ on $\partial U(j=1,2)$. 
(ii) The following equality holds:

$$
\sum_{j=1}^{2}\left(\int_{U} \sigma^{j, \varepsilon} \mathrm{d} x-\varepsilon \int_{\partial U} \frac{\partial \sigma^{j, \varepsilon}}{\partial \nu} \mathrm{d} S\right)=1
$$

In particular,

$$
\sum_{j=1}^{2} \int_{U} \sigma^{j, \varepsilon} \mathrm{d} x \leq 1
$$

We are now able to prove a uniform bound on $u_{1}^{\varepsilon}$ and $u_{2}^{\varepsilon}$. The proof is skipped, since it is analogous to those of the previous sections.

Lemma 5.4. There exists a positive constant $C$, independent of $\varepsilon$, such that

$$
\left\|u_{1}^{\varepsilon}\right\|_{L^{\infty}},\left\|u_{2}^{\varepsilon}\right\|_{L^{\infty}} \leq C .
$$

Next lemma will be used to give a uniform bound for $D u_{1}^{\varepsilon}$ and $D u_{2}^{\varepsilon}$.

Lemma 5.5. We have

$$
\left.\int_{U}\left(\gamma^{\varepsilon}\right)^{\prime}\right|_{\theta_{\varepsilon}^{1}} \sigma^{1, \varepsilon} \mathrm{d} x+\left.\int_{U}\left(\gamma^{\varepsilon}\right)^{\prime}\right|_{\theta_{\varepsilon}^{2}} \sigma^{2, \varepsilon} \mathrm{d} x \leq C,
$$

where $C$ is a positive constant independent of $\varepsilon$.

Proof. First of all, observe that condition (H5.1) implies that

$$
H_{j}(x, p)-D_{p} H_{j}(x, p) \cdot p \leq H_{j}(x, 0), \quad \text { for every }(x, p) \in \bar{U} \times \mathbb{R}^{n}, \quad j=1,2 .
$$

In the same way, the convexity of $\gamma$ implies

$$
\gamma^{\varepsilon}(s)-\left[\left(\gamma^{\varepsilon}\right)^{\prime}(s)\right] s=\gamma\left(\frac{s}{\varepsilon}\right)-\left[\left(\gamma^{\prime}\right)\left(\frac{s}{\varepsilon}\right)\right] \frac{s}{\varepsilon} \leq \gamma(0)=0 .
$$

Equation $(5.3)_{1}$ gives

$$
\begin{aligned}
0= & u_{1}^{\varepsilon}+H_{1}\left(x, D u_{1}^{\varepsilon}\right)+\left.\gamma^{\varepsilon}\right|_{\theta_{\varepsilon}^{1}}-\varepsilon \Delta u_{1}^{\varepsilon} \\
= & u_{1}^{\varepsilon}+D_{p} H_{1}\left(x, D u_{1}^{\varepsilon}\right) \cdot D u_{1}^{\varepsilon}-\varepsilon \Delta u_{1}^{\varepsilon}+H_{1}\left(x, D u_{1}^{\varepsilon}\right)-D_{p} H_{1}\left(x, D u_{1}^{\varepsilon}\right) \cdot D u_{1}^{\varepsilon} \\
& +\left.\gamma^{\varepsilon}\right|_{\theta_{\varepsilon}^{1}}-\left.\left(\gamma^{\varepsilon}\right)^{\prime}\right|_{\theta_{\varepsilon}^{1}} \theta_{\varepsilon}^{1}+\left.\left(\gamma^{\varepsilon}\right)^{\prime}\right|_{\theta_{\varepsilon}^{1}}\left(u_{1}^{\varepsilon}-u_{2}^{\varepsilon}\right)-\left.\left(\gamma^{\varepsilon}\right)^{\prime}\right|_{\theta_{\varepsilon}^{1}} \psi_{1} .
\end{aligned}
$$

Multiplying last relation by $\sigma^{1, \varepsilon}$, integrating and using (5.7) and (5.8)

$$
\begin{aligned}
\left.\int_{U}\left(\gamma^{\varepsilon}\right)^{\prime}\right|_{\theta_{\varepsilon}^{1}} \psi_{1} \sigma^{1, \varepsilon} \mathrm{d} x= & \int_{U}\left[H_{1}\left(x, D u_{1}^{\varepsilon}\right)-D_{p} H_{1}\left(x, D u_{1}^{\varepsilon}\right) \cdot D u_{1}^{\varepsilon}\right] \sigma^{1, \varepsilon} \mathrm{d} x \\
& +\int_{U}\left[\left.\gamma^{\varepsilon}\right|_{\theta_{\varepsilon}^{1}}-\left.\left(\gamma^{\varepsilon}\right)^{\prime}\right|_{\theta_{\varepsilon}^{1}} \theta_{\varepsilon}^{1}\right] \sigma^{1, \varepsilon} \mathrm{d} x \\
& +\int_{U}\left[\left(1+\left.\left(\gamma^{\varepsilon}\right)^{\prime}\right|_{\theta_{\varepsilon}^{1}}\right) u_{1}^{\varepsilon}+D_{p} H_{1}\left(x, D u_{1}^{\varepsilon}\right) \cdot D u_{1}^{\varepsilon}-\varepsilon \Delta u_{1}^{\varepsilon}-\left.\left(\gamma^{\varepsilon}\right)^{\prime}\right|_{\theta_{\varepsilon}^{1}} u_{2}^{\varepsilon}\right] \sigma^{1, \varepsilon} \mathrm{d} x \\
\leq & \int_{U} H_{1}(x, 0) \sigma^{1, \varepsilon} \mathrm{d} x \\
& +\int_{U}\left[\left(1+\left.\left(\gamma^{\varepsilon}\right)^{\prime}\right|_{\theta_{\varepsilon}^{1}}\right) u_{1}^{\varepsilon}+D_{p} H_{1}\left(x, D u_{1}^{\varepsilon}\right) \cdot D u_{1}^{\varepsilon}-\varepsilon \Delta u_{1}^{\varepsilon}-\left.\left(\gamma^{\varepsilon}\right)^{\prime}\right|_{\theta_{\varepsilon}^{1}} u_{2}^{\varepsilon}\right] \sigma^{1, \varepsilon} \mathrm{d} x .
\end{aligned}
$$


Analogously,

$$
\begin{aligned}
& \left.\int_{U}\left(\gamma^{\varepsilon}\right)^{\prime}\right|_{\theta_{\varepsilon}^{2}} \psi_{2} \sigma^{2, \varepsilon} \mathrm{d} x \leq \int_{U} H_{2}(x, 0) \sigma^{2, \varepsilon} \mathrm{d} x \\
& \quad+\int_{U}\left[\left(1+\left.\left(\gamma^{\varepsilon}\right)^{\prime}\right|_{\theta_{\varepsilon}^{2}}\right) u_{2}^{\varepsilon}+D_{p} H_{2}\left(x, D u_{2}^{\varepsilon}\right) \cdot D u_{2}^{\varepsilon}-\varepsilon \Delta u_{2}^{\varepsilon}-\left.\left(\gamma^{\varepsilon}\right)^{\prime}\right|_{\theta_{\varepsilon}^{2}} u_{2}^{\varepsilon}\right] \sigma^{2, \varepsilon} \mathrm{d} x .
\end{aligned}
$$

Summing up the last two relations and using (5.5) and (5.6)

$$
\begin{aligned}
& \left.\int_{U}\left(\gamma^{\varepsilon}\right)^{\prime}\right|_{\theta_{\varepsilon}^{1}} \psi_{1} \sigma^{1, \varepsilon} \mathrm{d} x+\left.\int_{U}\left(\gamma^{\varepsilon}\right)^{\prime}\right|_{\theta_{\varepsilon}^{2}} \psi_{2} \sigma^{2, \varepsilon} \mathrm{d} x \leq(2-i) u_{1}^{\varepsilon}\left(x_{0}\right)+(i-1) u_{2}^{\varepsilon}\left(x_{0}\right) \\
& \quad+\left\|H_{1}(\cdot, 0)\right\|_{L^{\infty}} \int_{U} \sigma^{1, \varepsilon} \mathrm{d} x+\left\|H_{2}(\cdot, 0)\right\|_{L^{\infty}} \int_{U} \sigma^{2, \varepsilon} \mathrm{d} x
\end{aligned}
$$

Thus,

$$
\begin{gathered}
\left.\int_{U}\left(\gamma^{\varepsilon}\right)^{\prime}\right|_{\theta_{\varepsilon}^{1}} \sigma^{1, \varepsilon} \mathrm{d} x+\left.\int_{U}\left(\gamma^{\varepsilon}\right)^{\prime}\right|_{\theta_{\varepsilon}^{2}} \sigma^{2, \varepsilon} \mathrm{d} x \leq \frac{2-i}{\alpha} u_{1}^{\varepsilon}\left(x_{0}\right)+\frac{i-1}{\alpha} u_{2}^{\varepsilon}\left(x_{0}\right) \\
+\frac{\left\|H_{1}(\cdot, 0)\right\|_{L^{\infty}}}{\alpha} \int_{U} \sigma^{1, \varepsilon} \mathrm{d} x+\frac{\left\|H_{2}(\cdot, 0)\right\|_{L^{\infty}}}{\alpha} \int_{U} \sigma^{2, \varepsilon} \mathrm{d} x \leq C,
\end{gathered}
$$

where we used (5.1), Lemma 5.3 and Lemma 5.4.

We can finally show the existence of a uniform bound for the gradients of $u_{1}^{\varepsilon}$ and $u_{2}^{\varepsilon}$.

Lemma 5.6. There exists a positive constant $C$, independent of $\varepsilon$, such that

$$
\left\|D u_{1}^{\varepsilon}\right\|_{L^{\infty}},\left\|D u_{2}^{\varepsilon}\right\|_{L^{\infty}} \leq C .
$$

\section{Proof. Step I: Bound on $\partial \mathrm{U}$}

As it was done in Section 2, we are going to construct appropriate barriers. For $\varepsilon$ small enough, assumption (H5.4) implies that

$$
\begin{cases}\Phi_{1}+H_{1}\left(x, D \Phi_{1}\right)+\gamma^{\varepsilon}\left(\Phi_{1}-\Phi_{2}-\psi_{1}\right)<\varepsilon \Delta \Phi_{1} & \text { in } U \\ \Phi_{2}+H_{2}\left(x, D \Phi_{2}\right)+\gamma^{\varepsilon}\left(\Phi_{2}-\Phi_{1}-\psi_{2}\right)<\varepsilon \Delta \Phi_{2} & \text { in } U\end{cases}
$$

and $\Phi_{1}=\Phi_{2}=0$ on $\partial U$. Therefore, $\left(\Phi_{1}, \Phi_{2}\right)$ is a sub-solution of (5.3). By the comparison principle, $u_{j}^{\varepsilon} \geq \Phi_{j}$ in $U, j=1,2$.

Let $d(x), \delta$, and $U_{\delta}$ be as in the Proof of Proposition 2.1. For $\mu>0$ large enough, the uniform bounds of $\left\|u_{1}^{\varepsilon}\right\|_{L^{\infty}}$ and $\left\|u_{2}^{\varepsilon}\right\|_{L^{\infty}}$ yield $v:=\mu d \geq u_{j}^{\varepsilon}$ on $\partial U_{\delta}, j=1,2$, so that

$$
\left\{\begin{array}{l}
v+H_{1}(x, D v)+\gamma^{\varepsilon}\left(v-v-\psi_{1}\right)-\varepsilon \Delta v=v+H_{1}(x, D v)-\varepsilon \Delta v \geq H_{1}(x, \mu D d)-\mu C \quad \text { in } U, \\
v+H_{2}(x, D v)+\gamma^{\varepsilon}\left(v-v-\psi_{2}\right)-\varepsilon \Delta v=v+H_{2}(x, D v)-\varepsilon \Delta v \geq H_{2}(x, \mu D d)-\mu C \quad \text { in } U .
\end{array}\right.
$$

Now, we have $\Phi_{j}=u_{j}^{\varepsilon}=v=0$ on $\partial U$. Also, thanks to assumption (H5.2), for $\mu>0$ large enough

$$
\begin{cases}v+H_{1}(x, D v)+\gamma^{\varepsilon}\left(v-v-\psi_{1}\right)-\varepsilon \Delta v \geq 0 & \text { in } U \\ v+H_{2}(x, D v)+\gamma^{\varepsilon}\left(v-v-\psi_{2}\right)-\varepsilon \Delta v \geq 0 & \text { in } U\end{cases}
$$


that is, the pair $(v, v)$ is a super-solution for the system (5.3). Thus, the comparison principle gives us that $\Phi_{j} \leq u_{j}^{\varepsilon} \leq v_{j}$ in $U_{\delta}$. Then, from the fact that $\Phi_{j}=u_{j}^{\varepsilon}=v=0$ on $\partial U$ we get

$$
\frac{\partial v}{\partial \nu}(x) \leq \frac{\partial u_{j}^{\varepsilon}}{\partial \nu}(x) \leq \frac{\partial \Phi_{j}}{\partial \nu}(x), \quad \text { for } x \in \partial U
$$

Hence, we obtain $\left\|D u_{j}^{\varepsilon}\right\|_{L^{\infty}(\partial U)} \leq C, j=1,2$.

\section{Step II: Bound on U}

Assume now that there exists $\widehat{x} \in U$ such that

$$
\max _{\substack{j=1,2 \\ x \in \bar{U}}} w_{j}^{\varepsilon}(x)=w_{1}^{\varepsilon}(\widehat{x}), \quad \quad \text { where } w_{j}^{\varepsilon}(x):=\frac{1}{2}\left|D u_{j}^{\varepsilon}\right|^{2}, \quad j=1,2 .
$$

By a direct computation one can see that

$$
2\left(1+\left.\left(\gamma^{\varepsilon}\right)^{\prime}\right|_{\theta_{1}^{\varepsilon}}\right) w_{1}^{\varepsilon}+D_{p} H_{1} \cdot D w_{1}^{\varepsilon}+D_{x} H_{1} \cdot D u_{1}^{\varepsilon}-\left.\left(\gamma^{\varepsilon}\right)^{\prime}\right|_{\theta_{1}^{\varepsilon}} D u_{1}^{\varepsilon} \cdot\left(D \psi_{1}+D u_{2}^{\varepsilon}\right)=\varepsilon \Delta w_{1}^{\varepsilon}-\varepsilon\left|D^{2} u_{1}^{\varepsilon}\right|^{2} .
$$

Multiplying last relation by $\sigma^{1, \varepsilon}$ and integrating over $U$

$$
\begin{aligned}
& 2 \int_{U} w_{1}^{\varepsilon} \sigma^{1, \varepsilon} \mathrm{d} x+\int_{U} D_{p} H_{1} \cdot D w_{1}^{\varepsilon} \sigma^{1, \varepsilon} \mathrm{d} x-\varepsilon \int_{U} \Delta w_{1}^{\varepsilon} \sigma^{1, \varepsilon} \mathrm{d} x+\int_{U} \varepsilon\left|D^{2} u_{1}^{\varepsilon}\right|^{2} \sigma^{1, \varepsilon} \mathrm{d} x \\
& +\int_{U} D_{x} H_{1} \cdot D u_{1}^{\varepsilon} \sigma^{1, \varepsilon} \mathrm{d} x+\left.\frac{1}{2} \int_{U}\left(\gamma^{\varepsilon}\right)^{\prime}\right|_{\theta_{1}^{\varepsilon}}\left[\left|D u_{1}^{\varepsilon}\right|^{2}+\left|D u_{1}^{\varepsilon}-D u_{2}^{\varepsilon}\right|^{2}-\left|D u_{2}^{\varepsilon}\right|^{2}\right] \sigma^{1, \varepsilon} \mathrm{d} x \\
& -\left.\int_{U}\left(\gamma^{\varepsilon}\right)^{\prime}\right|_{\theta_{1}^{\varepsilon}} D u_{1}^{\varepsilon} \cdot D \psi_{1} \sigma^{1, \varepsilon} \mathrm{d} x=0 .
\end{aligned}
$$

Then, using equation $(5.5)_{1}$ with $i=1$ and $x_{0}=\widehat{x}$

$$
\begin{aligned}
& \int_{U} w_{1}^{\varepsilon} \sigma^{1, \varepsilon} \mathrm{d} x+\int_{U} \varepsilon\left|D^{2} u_{1}^{\varepsilon}\right|^{2} \sigma^{1, \varepsilon} \mathrm{d} x-\left.\int_{U}\left(\gamma^{\varepsilon}\right)^{\prime}\right|_{\theta_{1}^{\varepsilon}} D u_{1}^{\varepsilon} \cdot D \psi_{1} \sigma^{1, \varepsilon} \mathrm{d} x \\
& +\int_{U} D_{x} H_{1} \cdot D u_{1}^{\varepsilon} \sigma^{1, \varepsilon} \mathrm{d} x+\left.\frac{1}{2} \int_{U}\left(\gamma^{\varepsilon}\right)^{\prime}\right|_{\theta_{1}^{\varepsilon}}\left[\left|D u_{1}^{\varepsilon}-D u_{2}^{\varepsilon}\right|^{2}-\left|D u_{2}^{\varepsilon}\right|^{2}\right] \sigma^{1, \varepsilon} \mathrm{d} x \\
& w_{1}^{\varepsilon}(\widehat{x})+\varepsilon \int_{\partial U} \frac{\partial \sigma^{1, \varepsilon}}{\partial \nu} w_{1}^{\varepsilon} \mathrm{d} S+\left.\int_{U}\left(\gamma^{\varepsilon}\right)^{\prime}\right|_{\theta_{2}^{\varepsilon}} w_{1}^{\varepsilon} \sigma^{2, \varepsilon} \mathrm{d} x=0
\end{aligned}
$$

which implies

$$
\begin{aligned}
w_{1}^{\varepsilon}(\widehat{x})-\left.\int_{U}\left(\gamma^{\varepsilon}\right)^{\prime}\right|_{\theta_{1}^{\varepsilon}} w_{2}^{\varepsilon} \sigma^{1, \varepsilon} \mathrm{d} x+\left.\int_{U}\left(\gamma^{\varepsilon}\right)^{\prime}\right|_{\theta_{2}^{\varepsilon}} w_{1}^{\varepsilon} \sigma^{2, \varepsilon} \mathrm{d} x \leq & \left.\int_{U}\left(\gamma^{\varepsilon}\right)^{\prime}\right|_{\theta_{1}^{\varepsilon}} D u_{1}^{\varepsilon} \cdot D \psi_{1} \sigma^{1, \varepsilon} \mathrm{d} x \\
& -\int_{U} D_{x} H_{1} \cdot D u_{1}^{\varepsilon} \sigma^{1, \varepsilon} \mathrm{d} x-\varepsilon \int_{\partial U} \frac{\partial \sigma^{1, \varepsilon}}{\partial \nu} w_{1}^{\varepsilon} \mathrm{d} S .
\end{aligned}
$$

Let now $\eta>0$ be a constant to be chosen later. Using Step I and Lemmas 5.3 and 5.5, thanks to Young's inequality

$$
\begin{aligned}
w_{1}^{\varepsilon}(\widehat{x})-\left.\int_{U}\left(\gamma^{\varepsilon}\right)^{\prime}\right|_{\theta_{1}^{\varepsilon}} w_{2}^{\varepsilon} \sigma^{1, \varepsilon} \mathrm{d} x+\left.\int_{U}\left(\gamma^{\varepsilon}\right)^{\prime}\right|_{\theta_{2}^{\varepsilon}} w_{1}^{\varepsilon} \sigma^{2, \varepsilon} \mathrm{d} x \leq & \left.\int_{U}\left(\gamma^{\varepsilon}\right)^{\prime}\right|_{\theta_{1}^{\varepsilon}}\left[\eta^{2} w_{1}^{\varepsilon}(\widehat{x})+\frac{\left\|D \psi_{1}\right\|_{L^{\infty}}^{2}}{2 \eta^{2}}\right] \sigma^{1, \varepsilon} \mathrm{d} x \\
& +\int_{U}\left[\eta^{2} w_{1}^{\varepsilon}(\widehat{x})+\frac{\left\|D_{x} H_{1}\right\|_{L^{\infty}}^{2}}{2 \eta^{2}}\right] \sigma^{1, \varepsilon} \mathrm{d} x+C \\
& \leq \eta^{2}(C+1) w_{1}^{\varepsilon}(\widehat{x})+C\left(1+\frac{1}{\eta^{2}}\right) .
\end{aligned}
$$


In the same way, considering the analogous of equation (5.9) for the function $w_{2}^{\varepsilon}$ (recalling that in (5.5) we chose $i=1$ ) we can obtain the following inequality :

$$
-\left.\int_{U}\left(\gamma^{\varepsilon}\right)^{\prime}\right|_{\theta_{2}^{\varepsilon}} w_{1}^{\varepsilon} \sigma^{2, \varepsilon} \mathrm{d} x+\left.\int_{U}\left(\gamma^{\varepsilon}\right)^{\prime}\right|_{\theta_{1}^{\varepsilon}} w_{2}^{\varepsilon} \sigma^{1, \varepsilon} \mathrm{d} x \leq \eta^{2}(C+1) w_{1}^{\varepsilon}(\widehat{x})+C\left(1+\frac{1}{\eta^{2}}\right)
$$

where we also used the fact that $\left\|w_{2}^{\varepsilon}\right\|_{L^{\infty}} \leq w_{1}^{\varepsilon}(\widehat{x})$. Summing inequalities (5.11) and (5.12) and choosing $\eta>0$ small enough the conclusion follows.

Next lemma gives a control of the Hessians $D^{2} u_{1}^{\varepsilon}$ and $D^{2} u_{2}^{\varepsilon}$ in the support of $\sigma^{1, \varepsilon}$ and $\sigma^{2, \varepsilon}$ respectively.

Lemma 5.7. There exists a positive constant $C$, independent of $\varepsilon$, such that

$$
\sup _{j=1,2} \int_{U} \varepsilon\left|D^{2} u_{j}^{\varepsilon}\right|^{2} \sigma^{j, \varepsilon} \mathrm{d} x \leq C .
$$

Proof. The bound of the Hessian of $D^{2} u_{1}^{\varepsilon}$ comes from identity (5.10), together with Lemma 5.6. The other bound can be obtained in an similar way.

We can finally prove the analogous of Lemma 2.2 .

Lemma 5.8. There exists a positive constant, independent of $\varepsilon$, such that

$$
\max _{\substack{j=1,2 \\ x \in \bar{U}}} \frac{\theta_{j}^{\varepsilon}(x)}{\varepsilon} \leq C, \quad \max _{\substack{j=1,2 \\ x \in \bar{U}}} \gamma^{\varepsilon}\left(\theta_{j}^{\varepsilon}(x)\right) \leq C .
$$

Proof. It will be enough to prove the second inequality, since the first one will follow by the definition of $\gamma^{\varepsilon}$. If the maximum is attained at the boundary, then

$$
\max _{\substack{j=1,2 \\ x \in \bar{U}}} \gamma^{\varepsilon}\left(\theta_{j}^{\varepsilon}(x)\right)=\max _{\substack{j=1,2 \\ x \in \partial U}} \gamma^{\varepsilon}\left(-\psi_{j}(x)\right)=0 .
$$

Otherwise, let us assume that there exists $x_{1} \in U$ such that

$$
\max _{j=1,2} \max _{x \in \bar{U}} \gamma^{\varepsilon}\left(\theta_{j}^{\varepsilon}\right)=\gamma^{\varepsilon}\left(\theta_{1}^{\varepsilon}\right)\left(x_{1}\right)>0, \quad \gamma^{\varepsilon}\left(\theta_{2}^{\varepsilon}\left(x_{1}\right)\right)=0
$$

Since $\gamma^{\varepsilon}$ is increasing and $\gamma^{\varepsilon}(z)>0$ if and only if $z>0$, we also have $\max _{x \in \bar{U}}\left(\theta_{1}^{\varepsilon}(x)\right)=\theta_{1}^{\varepsilon}\left(x_{1}\right)>0$. Evaluating the two equations in (5.3) at $x_{1}$ and subtracting the second one from the first one

$$
\begin{aligned}
\theta_{1}^{\varepsilon}\left(x_{1}\right)+\gamma^{\varepsilon}\left(\theta_{1}^{\varepsilon}\left(x_{1}\right)\right) & =\varepsilon \Delta u_{1}^{\varepsilon}\left(x_{1}\right)-\varepsilon \Delta u_{2}^{\varepsilon}\left(x_{1}\right)-H_{1}\left(x_{1}, D u_{1}^{\varepsilon}\left(x_{1}\right)\right)+H_{2}\left(x_{1}, D u_{2}^{\varepsilon}\left(x_{1}\right)\right)-\psi_{1}\left(x_{1}\right) \\
& \leq \varepsilon \Delta \psi_{1}\left(x_{1}\right)-H_{1}\left(x_{1}, D u_{1}^{\varepsilon}\left(x_{1}\right)\right)+H_{2}\left(x_{1}, D u_{2}^{\varepsilon}\left(x_{1}\right)\right)-\psi_{1}\left(x_{1}\right) \\
& \leq\left\|\Delta \psi_{1}(\cdot)\right\|_{L^{\infty}}+\left\|H_{1}\left(\cdot, D u_{1}^{\varepsilon}(\cdot)\right)\right\|_{L^{\infty}}+\left\|H_{2}\left(\cdot, D u_{2}^{\varepsilon}(\cdot)\right)\right\|_{L^{\infty}}+\left\|\psi_{1}(\cdot)\right\|_{L^{\infty}} \leq C,
\end{aligned}
$$

where we the last inequality follows from Lemma 5.6.

We now set for every $\varepsilon \in(0,1)$

$$
u_{j, \varepsilon}^{\varepsilon}(x):=\frac{\partial u_{j}^{\varepsilon}}{\partial \varepsilon}(x), \quad x \in \bar{U}, j=1,2 .
$$

The next lemma gives a uniform bound for $u_{1, \varepsilon}^{\varepsilon}$ and $u_{2, \varepsilon}^{\varepsilon}$, thus concluding the proof of Theorem 5.1. 
Lemma 5.9. There exists a positive constant $C>0$ such that

$$
\max _{\substack{j=1,2 \\ x \in \bar{U}}}\left|u_{j, \varepsilon}^{\varepsilon}(x)\right| \leq \frac{C}{\varepsilon^{1 / 2}} .
$$

Proof. If the above maximum is attained at the boundary, then

$$
\max _{\substack{j=1,2 \\ x \in \bar{U}}}\left|u_{j, \varepsilon}^{\varepsilon}(x)\right|=\max _{\substack{j=1,2 \\ x \in \partial U}}\left|u_{j, \varepsilon}^{\varepsilon}(x)\right|=0,
$$

since $u_{1, \varepsilon}^{\varepsilon}=u_{2, \varepsilon}^{\varepsilon}=0$ on $\partial U$. Otherwise, assume that there exists $\bar{x} \in U$ such that

$$
\max _{\substack{j=1,2 \\ x \in \bar{U}}}\left|u_{j, \varepsilon}^{\varepsilon}(x)\right|=\left|u_{1, \varepsilon}^{\varepsilon}(\bar{x})\right| .
$$

Differentiating (5.3) w.r.t. $\varepsilon$ we have

$$
\left\{\begin{array}{l}
\left(1+\left.\left(\gamma^{\varepsilon}\right)^{\prime}\right|_{\theta_{1}^{\varepsilon}}\right) u_{1, \varepsilon}^{\varepsilon}+D_{p} H_{1} \cdot D u_{1, \varepsilon}^{\varepsilon}-\left.\left(\gamma^{\varepsilon}\right)^{\prime}\right|_{\theta_{1}^{\varepsilon}} u_{2, \varepsilon}^{\varepsilon}+\left.\gamma_{\varepsilon}^{\varepsilon}\right|_{\theta_{1}^{\varepsilon}}=\varepsilon \Delta u_{1, \varepsilon}^{\varepsilon}+\Delta u_{1}^{\varepsilon} \quad \text { in } U, \\
\left(1+\left.\left(\gamma^{\varepsilon}\right)^{\prime}\right|_{\theta_{2}^{\varepsilon}}\right) u_{2, \varepsilon}^{\varepsilon}+D_{p} H_{2} \cdot D u_{2, \varepsilon}^{\varepsilon}-\left.\left(\gamma^{\varepsilon}\right)^{\prime}\right|_{\theta_{2}^{\varepsilon}} u_{1, \varepsilon}^{\varepsilon}+\left.\gamma_{\varepsilon}^{\varepsilon}\right|_{\theta_{2}^{\varepsilon}}=\varepsilon \Delta u_{2, \varepsilon}^{\varepsilon}+\Delta u_{2}^{\varepsilon} \text { in } U .
\end{array}\right.
$$

Let $\sigma^{1, \varepsilon}$ and $\sigma^{2, \varepsilon}$ be the solutions to system (5.4) with $i=1$ and $x_{0}=\bar{x}$. Multiplying $(5.13)_{1}$ and $(5.13)_{2}$ by $\sigma^{1, \varepsilon}$ and $\sigma^{2, \varepsilon}$ respectively, integrating by parts and adding up the two relations obtained we have

$$
u_{1, \varepsilon}^{\varepsilon}(\bar{x})=\sum_{j=1}^{2}\left(\int_{U} \Delta u_{j}^{\varepsilon} \sigma^{j, \varepsilon} \mathrm{d} x-\left.\int_{U} \gamma_{\varepsilon}^{\varepsilon}\right|_{\theta_{j}^{\varepsilon}} \sigma^{j, \varepsilon} \mathrm{d} x\right) .
$$

Thus,

$$
\left|u_{1, \varepsilon}^{\varepsilon}(\bar{x})\right| \leq \sum_{j=1}^{2}\left(\int_{U}\left|\Delta u_{j}^{\varepsilon}\right| \sigma^{j, \varepsilon} \mathrm{d} x+\int_{U}\left|\gamma_{\varepsilon}^{\varepsilon}\right|_{\theta_{j}^{\varepsilon}} \mid \sigma^{j, \varepsilon} \mathrm{d} x\right) .
$$

At this point, the proof can be easily concluded by repeating what was done in Section 2 showing relations $(2.12)-(2.15)$.

Acknowledgements. The authors are grateful to Craig Evans and Fraydoun Rezakhanlou for very useful discussions on the subject of the paper. We thank the anonymous referee for many valuable comments and suggestions. Diogo Gomes was partially supported by CAMGSD/LARSYS through FCT Program POCTI - FEDER and by grants PTDC/EEAACR/67020/2006, PTDC/MAT/69635/2006, and UTAustin/MAT/0057/2008. Filippo Cagnetti was partially supported by FCT through the CMU|Portugal program. Hung Tran was partially supported by VEF fellowship.

\section{REFERENCES}

[1] G. Barles and B. Perthame, Exit time problems in optimal control and vanishing viscosity method. SIAM J. Control Optim. 26 (1988) 1133-1148

[2] I. Capuzzo-Dolcetta and L.C. Evans, Optimal switching for ordinary differential equations. SIAM J. Control Optim. 22 (1984) $143-161$.

[3] F. Cagnetti, D. Gomes and H.V. Tran, Aubry-Mather measures in the nonconvex setting. SIAM J. Math. Anal. 43 (2011) 2601-2629.

[4] F. Camilli and P. Loreti, Comparison results for a class of weakly coupled systems of eikonal equations. Hokkaido Math. J. 37 (2008) 349-362.

[5] F. Camilli, P. Loreti, and N. Yamada, Systems of convex Hamilton-Jacobi equations with implicit obstacles and the obstacle problem. Commun. Pure Appl. Anal. 8 (2009) 1291-1302. 
[6] H. Engler and S.M. Lenhart, Viscosity solutions for weakly coupled systems of Hamilton-Jacobi equations. Proc. London Math. Soc. 63 (1991) 212-240.

[7] L.C. Evans and C.K. Smart, Adjoint methods for the infinity Laplacian partial differential equation. Arch. Ration. Mech. Anal. 201 (2011) 87-113.

[8] L.C. Evans, Adjoint and compensated compactness methods for Hamilton-Jacobi PDE. Arch. Ration. Mech. Anal. 197 (2010) $1053-1088$.

[9] D.A. Gomes, A stochastic analogue of Aubry-Mather theory. Nonlinearity 15 (2002) 581-603.

[10] H. Ishii and S. Koike, Viscosity solutions for monotone systems of second-order elliptic PDEs. Commun. Partial Differ. Equ. 16 (1991) 1095-1128.

[11] K. Ishii and N. Yamada, On the rate of convergence of solutions for the singular perturbations of gradient obstacle problems. Funkcial. Ekvac. 33 (1990) 551-562.

[12] P.L. Lions, Generalized solutions of Hamilton-Jacobi equations, Research Notes in Mathematics. Pitman (Advanced Publishing Program), Boston, Mass. 69 (1982).

[13] P.L. Lions, G. Papanicolaou and S.R.S. Varadhan, Homogenization of Hamilton-Jacobi equations, Preliminary Version, (1988).

[14] H.V. Tran, Adjoint methods for static Hamilton-Jacobi equations. Calc. Var. Partial Differ. Equ. 41 (2011) $301-319$. 